\title{
Las estrategias nacionales de desarrollo y la integración de los Países Andinos ${ }^{* * *}$
}

Las distintas motivaciones de los países para participar en un proceso de integración económica, la naturaleza y la forma que ćste asume, el apoyo que suscita y el grado cle conflicto que provoca entre los Estados participantes configuran un conjunto de tópicos claves en el campo de las relaciones económicas internacionales. Estos aspectos, por otra parte, contribuyen decisivamente a determinar las perspectivas de la propia integración y configuran el contexto en que debe situarse la discusión acerca del crucial problema de la distribución de beneficios y costos del proceso.

Pese a la importancia de este tema, son escasos los estudios que los abordan en forma analítica y comprensiva a la vez. La tarea es compleja, porque requiere hacer explícita la estrecha vinculación existente entre aspectos puramente económicos y variables políticas, sociales o ideológicas relativas a los sistemas de organización social que imperan en los distintos paises. En este sentido, este tema se relaciona también con el problema del grado en que es viable un proceso de integración económica entre países con diferentes sistemas y orientaciones socio-políticas.

Este trabajo se propone analizar tales aspectos para el caso específico de la integración andina***. Su vitalidad demuestra inequivocamente que han sido más los puntos de acuerdo que las divergencias entre las naciones de la Subregión. Basta señalar que en los cinco años transcurridos desde la iniciación del proceso, éste ha sobrevivido al cambio de casi tres gobiernos por país, en promedio, pese a

*E1 autor desea agradecer los comentarios y sugerencias que lc formularon sus colegas de CEPLAN. Sin embargo, los juicios que aquí se vicrten son de su exclusiva responsabilidad.

*Este trabajo forma parte de un libro sobre la realidad y perspectivas del proceso de integración subregional que el Centro Interuniversitario de Desarrollo Andino (CINDA) publicará en fecha próxima. El presente articulo se terminó de escribir a fines de 1975, mientras el autor se desempeñaba también como Coordinador del Árca Económica de CINDA.

*Un trabajo sobre este tema, sin embargo, en un proceso del dinamismo de la integración andina, no puede pretender ser válido en todo momento del tiempo. En efecto, después de terminar de cscribirse este artículo en diciembre de 1975, dicho proceso ha sufrido algunos cambios que no han sido posible incorporar en cste documento. Por lo tanto, debe advertirse que cste estudio sólo pretende reflejar las tendencias fundamentales que sc apreciaban en el momento indicado. 
que algunos regímenes sustentaban posiciones diametralmente opuestas a las de sus antecesores. No obstante, es necesario desarrollar un marco de referencia teórico que permita analizar en forma conjunta todas las variables que determinan el grado de adhesión de los Estados signatarios del Acuerdo de Cartagena.

Los supuestos en que se funda este trabajo son, en primer término, que la integración es un proceso negociado y esencialmente dinámico, donde los participantes definen bases de vinculación entre ellos a partir del descubrimiento de que ciertos intereses nacionales son comunes $^{1}$. En este sentido, la integración tiene desde el punto de vista de cada país el carácter de un instrumento que permite alcanzar mejor ciertas metas nacionales prioritarias; rara vez es un objetivo o un valor en sí mismo. ${ }^{2}$

En segundo lugar, por ser la integración un proceso dotado de una dinámica propia va asumiendo una existencia relativamente autónoma, con órganos y mecanismos que le son específicos, todos los cuales configuran lo que llamaremos un modelo particular de integración. Por lo tanto las principales coincidencias o discrepancias de intereses nacionales, las cuales condicionan en última instancia el grado de adhesión de cada país al proceso o la evolución que éste experimenta, pueden plantearse directamente en relación con ese modelo particular antes que como diferencias sólo entre los intereses nacionales.

Por último, en la definición de los intereses de cada país influyen tanto los costos y beneficios económicos de los diferentes mecanismos de integración, como la "valoración" ideológica o política que se haga de ellos. Distinguir una dimensión de otra resulta clave para entender el estado del proceso y sus perspectivas. Así, por ejemplo, el empleo de cierto mecanismo, como la programación industrial, puede acarrear beneficios económicos a un país, pero resultarle "poco deseable" política o ideológicamente porque contradice la estrategia global de desarrollo que se ha fijado, que en este caso

${ }^{1}$ Estos intereses pueden ser cconómicos o propiamente políticos, es decir referirsc a la consolidación de un determinado sistema de gobierno como, por ejemplo, el parlamentario vis à vis un régimen de otro tipo. Este aspecto fue de gran importancia en la integración curopea occidental que surgió después de la Segunda Guerra Mundial. Véanse por ejemplo los artículos de Valdés G., "El sistema político latinoamericano como condición de la integración" y Rioseco A. y B. Nun, "La integración andina, su carácter político, presupuestos y consecuencias", ambos en CINDA, et al., 1973.

${ }^{2}$ Los gobiernos de diversos países de la Subregión han reconocido de manera muy explícita el carácter instrumental que atribuyen a la integración. Véanse, a título de ejemplo, JUNAC, "Informe final del Primer Seminario Inter-regional sobre Planificación Integrada de los Países Miembros", y los artículos de Caballero C. (1975), e Iñiguez J. y M. Vega-Centeno (1975). 
podría ser dejar sólo al mercado la decisión sobre la localización de actividades productivas y asignación de recursos. Naturalmente, las variables económicas y político-ideológicas pueden actuar en el sentido de reforzarse o contraponerse, y no siempre son fáciles de discernir ${ }^{3}$. El hecho es que ambas, conjuntamente, influirán sobre la adhesión que los países presten a cada programa de integración. Dado que la estrategia nacional de desarrollo determina implícitamente por lo menos la importancia relativa que concede a cada programa de integración y a sus efectos, permite también hacer inferencias con respecto al grado de adhesión de ese país al proceso global.

El capítulo inicial de este trabajo describe el carácter general del Acuerdo de Cartagena y su relación original y actual con los diferentes tipos de estrategias nacionales de desarrollo que siguen las seis naciones que lo suscribieron. Tales estrategias son examinadas en el capítulo II, estudiándose en el siguiente los efectos económicos probables de los principales programas o mecanismos de la integración sobre cada país. El capítulo IV está destinado a evaluar, a la luz de los intereses económicos e ideológicos de cada país, los grados de conflicto y armonía que pueden suscitarse entre los diferentes Estados en el transcurso del proceso. Termina el trabajo con un breve análisis de las perspectivas y principales desafíos que afronta la integración andina al promediar la década del setenta.

\section{I. - LOS MODELOS ANDINOS DE INTEGRACIÓN Y DESARROLLO}

En este capítulo se analizarán dos aspectos: primero, la relación más específica entre las estrategias nacionales de desarrollo y un proceso de integración; segundo, las características básicas del esquema andino, en la forma en que fueron definidas en el Acuerdo de Cartagena, que corresponde implícitamente a cierta estrategia específica de desarrollo.

\section{Relaciones entre estrategias de desarrollo e integración}

La firma de un tratado como el Acuerdo de Cartagena refleja, estrictamente, una coincidencia o consenso mínimo de los países que lo suscriben acerca de ciertos objetivos y respecto de los mecanismos

\footnotetext{
"En particular, es raro que los gobiernos reconozcan que las posiciones que asumen se fundan en razones ideológicas o políticas.
} 
que juzgan aceptable utilizar para su consecución ${ }^{4}$. El Acuerdo explicita, por lo tanto, los intereses nacionales que son comunes a ese grupo, los cuales responden no sólo a consideraciones de índole económica, sino también a valores políticos o ideológicos, que se expresan en términos de la "aceptabilidad" de los mecanismos seleccionados para el logro de dichos objetivos. Naturalmente, esos intereses se refieren a un área limitada del total de los intereses nacionales. Se vinculan, en efecto, a los más directamente asociados a sus relaciones con los demás países y, especialmente, al intercambio económico con ellos. ${ }^{5}$

Tanto los objetivos nacionales, o la percepción de los principales obstáculos para el desarrollo, como cl sistema económico global y las políticas que deben aplicarse surgen de lo que hemos denominado las estrategias nacionales de desarrollo de los países, razón por la cual éstas constituyen un elemento clave del análisis.

Una estrategia de desarrollo - esté o no expresada en forma explícita- abarca tres aspectos fundamentales, estrechamente interrelacionados. En primer lugar, los objetivos globales, no sólo económicos, sino sociales, políticos y culturales del país. En otras palabras, el estilo de sociedad misma que se aspira a construir y, en particular, la organización económica en que ella debe sustentarse. En este campo se sitúan las decisiones relativas al papel del Estado y del sector privado en la cconomía en general, y sobre la planificación y el mercado en particular. En segundo término, comprende los principales grupos o actores sociales y políticos que plantean y sostienen esos objetivos y ese modelo de sociedad, los cuales se benefician eventualmente en mayor medida con su aplicación. En tercer lugar, la estrategia pública contempla el conjunto de medidas técnicas o políticas que se estiman más adecuadas para alcanzar esas metas.

En la práctica, la estrategia de desarrollo de un país no corresponde ni a una decisión completamente exógena a la sociedad misma -impuesta por el Estado o Gobierno, por ejemplo- ni tampoco a una decisión en que efectivamente participe toda la sociedad. Constituye un compromiso de naturaleza dinámica entre los diversos grupos sociales, políticos y económicos. El Estado juega el rol fundamental de ser una especie de garantía de ese compromiso y su principal ejecutor, interna y externamente; en esa medida representa los

'Un cstudio que analiza el proceso de integración andina con un enfoque en cierta manera similar al utilizado en esta sección es el de E. Camacho (1975).

${ }^{\circ}$ En otras palabras, la firma de un acuerdo de integración y su implementación no tiene por qué implicar ese consenso con respecto a otros intereses nacionales -como el rol del Estado en la cconomía, la distribución del ingreso o el sistema de propiedad de las empresas-, que por mucha importancia que revistan no se vinculan tan directamente a materias que afectan la relación entre los Paises miembros. 
intereses del conjunto de grupos sociales que coexisten en el país ${ }^{i}$. Pero el Estado no es un ente exógeno o independiente que representa un interés nacional abstracto, sino que el de los grupos mencionados, de acuerdo a la distribución del poder de cada uno de ellos en la sociedad que el Estado contribuye normalmente a legitimar. Así se interpretará en este trabajo el concepto de interés nacional y la posición que asumen los Estados nacionales en las negociaciones que comprende un proceso de integración.

Las estrategias de desarrollo tienen por lo común más permanencia en el tiempo que los gobiernos que controlan el poder del Estado, aunque ciertamente son influidas por las posiciones de los gobiernos. Aquellas reflejan los objetivos de más largo plazo que se han fijado el país o, más precisamente, los grupos dominantes dentro de éste. Para que la estrategia cambie en términos significativos, se requiere entonces que se produzca una modificación en la estructura del poder y que emerjan como dominantes grupos con intereses diferentes de los que hasta entonces ocupaban esa posición. Los gobiernos, en cambio, son normalmente menos estables porque reflejan la correlación de fuerzas respecto de asuntos más contingentes.

No obstante estas salvedades, es obvio que resulte extremadamente difícil mantener en forma rigurosa esas interpretaciones en la práctica o detectar cuándo deben ser modificadas. Así, por ejemplo, en ciertos momentos la estrategia de desarrollo de un país puede ser "impuesta" por un gobierno y, en consecuencia, podría decirse que ella no está representando los valores e intereses de la colectividad nacional, sino sólo los de la élite gobernante.

En esa medida debería hablarse, en cierto sentido, de la estrategia de desarrollo (o los valores e intereses) impulsada por determinado gobierno y no por "el país". Al llegar a este nivel, se observa claro lo complejo y lo necesario que resulta tanto precisar la definición de los términos empleados como interpretar cuidadosamente los juicios emitidos y las conclusiones alcanzadas.

Las características estructurales de la economía de un país y el diagnóstico o percepción de ellas por parte de los grupos dominantes en la sociedad son elementos que gravitan de manera decisiva en la definición de una estrategia nacional. Entre las primeras se encuentran factores como el nivel de desarrollo del país, su tasa de crecimiento, la disponibilidad de recursos, la estructura de comercio exterior, etc.

Un determinado modelo o esquema de integración se origina a partir de la interacción del conjunto de elementos que comprenden las estrategias de desarrollo de cada país. En la medida que va más

'Poulantzas, 1970. 
allá de un simple aumento, y por cualquier medio, de sólo el intercambio comercial dentro del área involucrada implica la especificación de cómo intensificar ese intercambio y promover todo un proceso de desarrollo conjunto. El esquema de integración debe contemplar, por lo tanto, un conjunto de mecanismos específicos y políticas comunes de los países, e instituciones destinadas a implantarlos y velar por su cumplimiento.

La forma específica que asuma el proceso de integración no es independiente de las estrategias de desarrollo de los países que lo impulsan. Esto no significa ni remotamente, sin embargo, que ambos procesos deban coincidir para todas o cada una de las naciones involucradas. En primer lugar, porque al momento de comprometerse en determinada forma específica o modelo de integración, los Estados miembros negocian y transan entre ellos, llegando a un acuerdo que refleja un "común denominador" de sus respectivos intereses ${ }^{7}$. Segundo, ese modelo surge en determinado momento del tiempo, bajo circunstancias históricas externas o internas muy concretas. Por lo tanto, aun suponiendo que en esa coyuntura el modelo adoptado hubiese sido congruente con las estrategias de desarrollo nacionales, éstas normalmente van evolucionando en distinta dirección o con diferente rapidez en cada país. La implementación de cualquier modelo de integración demora algún tiempo y configura un proceso que es más difícil de modificar que la estrategia de un país considerado aisladamente, porque la revisión de un acuerdo colectivo requiere cierto consenso. Es posible entonces que en una fase más avanzada ese modelo no corresponda a los intereses nacionales de alguno y tal vez de ninguno de los países miembros. En la medida que ello ocurra, el tipo o modelo de integración definido inicialmente se iría alternando porque -como se desprende de lo anterior- este último no es un proceso exógeno a las estrategias de desarrollo de los países.

En particular, los mecanismos o programas concretos que comprende un esquema de integración como el andino surgen de un consenso básico de las naciones participantes, pero no son, necesariamente, los "optimos" para cada uno de ellos aisladamente; en otras palabras, dicho esquema no siempre coincide con ciertos intereses nacionales específicos.

${ }^{\tau}$ Ya se señaló que en el caso andino ese compromiso quedó formalizado en el propio Acuerdo de Cartagena. La Junta ha procurado ir más allá, haciendo explicito el modelo de desarrollo conjunto que estaría implícito en los términos del Acuerdo. Esto se expresó en el documento Bases Generales para una Estrategia Subregional de Desarrollo, publicado en 1972. Sin embargo, a pesar de su trascendencia del tema que aborda, no ha sido posible lograr el consenso necesario sobre esas "Bases" para permitir que sea aprobado por todos los países como documento programático oficial. 
¿Existe alguna fórmula para evaluar en qué medida la aplicación de determinado mecanismo es más o menos aceptable para un país? La tesis que se plantea aquí es que a este respecto hay que considerar dos criterios básicos: por una parte, el costo o beneficio puramente económico y, por otra, el valor politico-ideológico que se asigne a los mecanismos o programas de que se trate, en virtud cle su discrepancia o coincidencia con el tipo de políticas nacionales que permiten implementar una determinada estrategia de desarrollo de un país.

Una de las formas de visualizar la relación entre estos dos criterios es planteando que los costos o beneficios económicos de la integración deben ser adecuadamente "ponderados" por el "valor político o ideológico" que implica obtenerlos. Estas ponderaciones, que en algunos casos podrían ser incluso negativas, diferirán por lo común de un país a otro, dependiendo de sus estrategias nacionales de desarrollo. Así, entonces, los efectos de un mismo mecanismo de integración -por ejemplo, una modificación de los aranceles externos que genere idénticas consecuencias económicas en dos países que tengan similar estructura productiva y de comercio- pueden ser percibidos como un "beneficio" por uno y un "costo" por otro.

Es claro, sin embargo, que cada país no puede pretender que el uso que se da a los diferentes mecanismos de integración le satisfaga plenamente, ni aplicar sólo los instrumentos y programas que le reporten beneficios. Su adhesión al proceso se explica porque ha estimado que éste le permite obtener un efecto global neto de signo favorable; sin embargo, es necesario establecer cuál es la ponderación relativa que concede ese país a los costos y beneficios que acarrea cada aspecto que comprende la integración: la desgravación arancelaria interna, el arancel externo común, la programación sectorial, la armonización de otras políticas (especialmente respecto al capital extranjero), etc.

Para establecer la adhesión o reservas de un país a determinado esquema de integración conviene analizar en forma más desagregada la armonía o conflicto de sus intereses en relación a los reflejados en ese esquema. Por lo tanto, en la próxima sección se analizan los

${ }^{8}$ Dicho plantcamiento pone de relieve las limitaciones del estudio de la distribución de costos y beneficios cconómicos de la integración entre países cuando no se considera el contexto que se destaca en este trabajo. En términos de la teoría neoclásica del bienestar, los costos y beneficios económicos para un país sólo pueden determinarse en relación a la "función de bienestar social", que refleja los "juicios de valor" de la comunidad de que se trata. El problema es que no existe una sola función de bienestar para toda la Subregión Andina, sino que es preciso trabajar con la de cada país. En cicrto sentido, lo que aquí se intenta es hacer explicita esta última, a través del expediente de asociarla con la respectiva estrategia de desarrollo. 
principales rasgos del modelo andino y de las estrategias nacionales de los países, estableciéndose comparaciones entre unos y otros.

\section{El modelo de integración andina}

En primer término, en su concepción general el proceso de integración andina definido a fines de la década del sesenta, se deriva principalmente de un diagnóstico común sobre el origen del estancamiento de los procesos de industrialización de los países de desarrollo intermedio de América Latina. Dicho fenómeno se atribuyó a las limitaciones del modelo de sustitución nacional indiscriminada de importaciones que hacía imposible generar un grado aceptable de eficiencia en la producción interna, un aprovechamiento adecuado de economías de escala y un desarrollo tecnológico más autónomo. Esto se observa especialmente a nivel de ciertas industrias estratégicas o básicas -como las del acero, la petroquímica, la metal mecánica y otras-, varias de las cuales habían surgido al amparo de la protección del Estado.

En segundo lugar, la integración andina fue definida como un instrumento para que las naciones involucradas expandieran y diversificaran sus exportaciones, reduciendo su dependencia de unas pocas materias primas. La consecución de este objetivo se había revelado para estos países como una empresa muy ardua dentro de la ALALC, porque en ella el grueso de las exportaciones, especialmente de bienes manufacturados, era controlado por las naciones grandes (Brasil, México y Argentina). Más difícil aún resultaba a cada país andino aisladamente penetrar los mercados de las economías industrializadas, protegidos por restricciones y tarifas que se hacen más gravosas a medida que aumenta el grado de elaboración de los productos ${ }^{9}$.

En tercer lugar, a un nivel relativo a la estrategia global del proceso integracionista, debe señalarse que si bien al sector privado $-\mathrm{y}$, en consecuencia, también al mercado- se le reservó un papel muy importante en cuanto a la generación de comercio subregional, el esquema andino otorga un rol preponderante a la planificación y al Estado. A éste corresponden las principales decisiones e iniciativas destinadas a configurar el marco para el funcionamiento adecuado de las empresas privadas en el mercado, situación que contrasta con lo que sucede en la ALALC, donde la iniciativa se reserva a los empresarios privados quienes se reúnen para negociar los denominados Acuerdos de Complementación y proponer rebajas arancelarias. En cambio, en el modelo andino, los países miembros se comprometieron

'Además, hay otras razones - como la presencia de corporaciones transnacionales- que hacen a esos mercados muy poco competitivos e inestables. 
a una programación conjunta de industrias en sectores claves, a una rebaja predeterminada de aranceles regionales y a la imposición de un arancel externo común. La iniciativa la asumen los respectivos Estados, debiendo las empresas existentes en cada país negociar con las autoridades nacionales la implementación de tales medidas en el plano interno.

Finalmente, el modelo andino se caracteriza por una clara conciencia de la importancia de la distribución equitativa de los beneficios y costos del proceso entre las naciones participantes. Esta es una lección que se desprende en gran parte de la experiencia de ALALC, donde se había comprobado que, en ausencia de mecanismos especiales, aquellos países de menor desarrollo relativo inicial tendían a verse menos beneficiados en términos comparativos. ${ }^{10}$

El modelo de integración andina aparece entonces como un proceso relativamente dirigido o planificado, característica que se derivó de las estrategias que aplicaban hacia fines de los años sesenta los principales países andinos. Postulaban ellas el establecimiento de una economía "mixta" que implicaba en alguna medida reformar los aspectos más "capitalistas" del patrón de desarrollo seguido hasta entonces. Consultaban para tal efecto una participación más activa del Estado y la planificación de la actividad económica.

Con el objeto de llegar a la configuración de un área integrada, el modelo andino contemplaba tres tipos de medidas principales: a) la liberación gradual - pero predeterminada y automática- del comercio entre los países miembros; b) la adopción de varias políticas comunes, especialmente respecto de aranceles externos y tratamiento del capital extranjero; y c) la asignación planificada de industrias. ${ }^{11}$

Respecto al primer mecanismo, el Acuerdo de Cartagena contempla la apertura del comercio recíproco o regional en virtud de un Programa de Liberación (PL) que: i) implica una reducción predeterminada y automática de aranceles, de 10 puntos porcentuales cada año, y ii) es, en última instancia, generalizado para todos los productos, aunque las modalidades de liberación difieren entre tipos de bienes y países. Bolivia y Ecuador tienen ciertas ventajas en términos de plazos más amplios, mantención por algún tiempo de aranceles superiores a los de los demás países y liberación más rápida por parte de sus asociados de algunos productos que esas naciones de menor desarrollo relativo exportan. En lo que se refiere a diferencias

${ }^{10}$ Una demostración indirecta, pero evidente de esta tendencia se aprecia al observar que de los 20 Acuerdos de Complementación suscritos en cl seno de la ALALC, Brasil participa en 17 (el $85 \%$ ) y Argentina y México en 14 (el 70\%), mientras que Ecuador no participa en ninguno, Bolivia en uno, Perú y Colombia en 2, y Chile alcanza un máximo de $6(30 \%)$. Véase INTAL (1975).

${ }^{11}$ Una descripción detallada de estos instrumentos se cncucntra en A. Aninat (1975), y R. Ffrench-Davis (1975). 
en función de los tipos de productos, debe señalarse que la liberación es abrupta para los bienes no producidos inicialmente en la región y más lenta para aquellos que afectan en forma intensa las industrias ya instaladas, asumiendo modalidades especiales para los involucrados en los PSDI.

Con respecto a la adopción de políticas comunes, una de las más cruciales se refiere al AEC, que define el margen de protección a la producción regional. Ya se adoptó un arancel externo mínimo común (AEMC) en 1970, y se espera iniciar gradualmentc la aplicación del AEC, de modo de alcanzar ciertos niveles predetcrminados en 1980. La elaboración del AEC definitivo constituye una de las tareas más complejas y conflictivas dentro del proceso de integración subregional. ${ }^{12}$

Cualquiera sea el AEC que en definitiva se apruebe, afectará en forma diferente a cada pais, dependiendo de su estructura productiva y de consumo, apertura al comercio exterior, nivel de aranceles iniciales, etc., y de las preferencias político-ideológicas que predominen en él. En tal sentido, la incidencia de este instrumento sobre la distribución de los beneficios de la integración entre países es meridiana. En general, lo más probable es, por una parte, que el AEC definitivo resulte inferior al nivel promedio que tenían los países inicialmente, lo cual deriva tanto de la mayor eficiencia productiva que debe resultar de la constitución de un mercado más amplio, como del objetivo generalizado de fomentar las exportaciones hacia terceros países. Por otra parte, la reducción de los aranccles que recaen sobre los bienes de consumo tendría que ser mucho mayor que la de aquellos que recaen sobre los bienes de capital, los cuales deberían incluso, en el caso de algunos países, ser alzados. El objetivo explicito de propender a una mayor producción regional de estos últimos requiere una mayor protección frente a la competencia del resto del mundo, lo cual supone invertir la tendencia tradicional de imponer tarifas bajas y conceder una serie de franquicias y excepciones a la importación de bienes de capital.

La segunda política común importante se refiere al tratamicnto al capital extranjero. Responde ella a un criterio tanto de cficiencia como de equidad, tratando de impedir que la localización de empresas en determinado país obedezca a criterios económicos (como menores costos relativos de producción reales) y no a políticas más favorables en ese país (como podría ser un trato impositivo especial). Procura asimismo que los esfuerzos para formar un mercado común exporte

12Dicho arancel comenzaría a aplicarse el 31 de diciembre de 1976, después de negociaciones que deben llevarse a cabo a partir de una propuesta presentada por la Junta a la Comisión del Acuerdo. 
beneficios equitativos para las naciones que impulsan este proceso y no a grupos o países de fuera de la región. ${ }^{13}$

Finalmente, el tercer mecanismo general que contempla el esquema de integración andino se refiere a los Programas Sectoriales de Desarrollo Industrial (PSDI), que implican sustraer de las empresas privadas -especialmente extranjeras- la decisión de dónde (en que país) localizar la producción de ciertos bienes. La idea fundamental es usar dichos programas con un propósito redistributivo, asegurando la instalación de una cuota razonable de nuevas empresas en los países de menor desarrollo relativo.

\section{II. - ACTITUdES POLÍtICO-IDEOLÓGICAS FRENTE A LA INTEGRACIÓN:}

LAS ESTRATEGIAS NACIONALES DE DESARROLLO

Como se señaló al comienzo, el apoyo o rechazo de cada país al modelo andino de integración depende en parte de factores político-ideológicos. Tienen ellos su origen en la estrategia de desarrollo que el país ha adoptado; ésta refleja, a su vez, el poder relativo de los grupos sociales en el sistema socio-económico que estructuran, así como el "valor" que los grupos dominantes atribuyen al uso de las diferentes políticas o instrumentos que contempla el modelo. Se plantea en esta sección una síntesis de los tres tipos principales de estrategias nacionales de desarrollo que se perfilan en el Grupo Andino. Con estos antecedentes y teniendo en cuenta además los efectos económicos "puros" de la integración en cada país - que serán examinados separadamente en el próximo capítulo- se procurará visualizar las áreas de conflicto y armonía de intereses entre cada país y el proceso integracionista.

Al promediar la década del setenta, las principales estrategias de desarrollo explícitas o implícitas de los países andinos podían catalogarse en tres tipos; una de libre mercado o de carácter neoliberal; y otra de tipo más planificado y una tercera de naturaleza mixta que sigue más la estrategia de fines de la década del sesenta.

Lo primero que cabe destacar es que esta clasificación, como todas, presenta algún grado de arbitrariedad, ignorando que también dentro de cada tipo existen variaciones y matices. En segundo lugar, no es fácil clasificar a los países, especialmente debido a lo poco explícito de las propias estrategias de desarrollo que siguen ${ }^{14}$. Finalmente, esa

${ }^{13}$ Salgado (1973) .

${ }^{14 E n ~ e f e c t o, ~ e l l a s ~ r a r a ~ v e z ~ s e ~ v e n ~ r e f l e j a d a s ~ e n ~ l a s ~ c o n s t i t u c i o n e s ~ p o l i ́ t i c a s ~ d e ~}$ los países, declaraciones de principios de los Gobiernos o planes nacionales de desarrollo. 
tipología no debe considerarse como algo estático. Ya se destacó antes que las estrategias van evolucionando a través del tiempo e, incluso, experimentan en algunos casos fuertes modificaciones. Por otra parte, algo que es calificado de tradicional hoy día, tal vez haya sido muy heterodoxo hace diez años. En este sentido, además, influye no sólo el contexto nacional en que se formulan las diferentes estrategias, sino también, y muy especialmente, factores de orden externo. ${ }^{15}$

Por último, siempre es difícil y riesgoso colocar nombres para distinguir las diferentes estrategias porque resulta casi imposible cvitar que a las denominaciones utilizadas se les confiera una connotación valorativa o que se las critique en razón a que pudo haberse empleado otras. Sin embargo cabe sólo reiterar que en el presente trabajo no se otorga a ninguna de ellas ni un sentido apologético ni peyorativo, sino uno meramente operacional.

\section{La estrategia tradicional}

Hemos denominado tradicional a la estrategia que corresponde esencialmente a la que seguían la mayoría de los países andinos a fines de la década de 1960, cuando se firmó el Acucrdo de Cartagena. Es la que, con algunas variantes, han continuado implementando Bolivia, Colombia, Ecuador y Venezuela.

En dicha estrategia, el Estado y el sector privado, en especial el constituido por los empresarios del sector manufacturero en el caso de los países relativamente más adelantados, juega un rol de importancia relativamente similar. Dentro del último sector, los grupos que más entusiasta apoyo brindan a la integración están conformados por los exportadores potenciales al mercado ampliado, mientras que los más recelosos son los productores de sustitutos de importaciones, aunque a veces son los mismos grupos quienes actúan como importadores y exportadores. El Estado asume un rol medianamente activo respecto a la integración en el sentido de sostencr posiciones muy condicionadas por los intereses de los grupos más poderosos del sector privado que sc ven perjudicados, mientras también debe considerar otras presiones, que se reflejan en una búsqueda por reducir los precios de muchos artículos manufacturados y por elevar la eficiencia de la actividad manufacturera. ${ }^{16}$

Un ejemplo que ilustra este aspecto lo proporciona la incorporación de Venezuela al Pacto Andino. En efecto, en una primera etapa el

\footnotetext{
${ }^{15}$ Para un interesante análisis sobre esta materia, véase Salgado, G., "Notas sobre la integración andina y su contexto internacional".

${ }^{10}$ Melo (1973) proporciona antecedentes sobrc estc aspecto para el caso de Colombia.
} 
sector industrial privado se opuso vigorosamente a que el país participara en el proceso de integración subregional. Y aunque el Gobierno presionaba en el sentido contrario, sólo vino a imponer su criterio tres años después de haberse suscrito el Acuerdo de Cartagena. El ingreso de Venezuela en 1973 parece haberse debido en gran parte a que el empresariado nacional fue modificando con el transcurso del tiempo la apreciación que tenía acerca de la magnitud de los riesgos que la competencia de las importaciones andinas entrañaba. ${ }^{17}$

Desde el punto de vista de las políticas económicas vinculadas a la integración, la estrategia tradicional otorga prioridad a la industrialización, a través del establecimiento de un nivel intermedio de protección arancelaria, buscando una sustitución de importaciones a nivel regional, lo que implica procurar un aumento de las exportaciones andinas. ${ }^{18}$

En general, los países que siguen esta estrategia tienen una posición político-ideológica relativamente favorable a los cuatro programas básicos que ha ido implementando la Comisión del Acuerdo de Cartagena, y otorgan a todos ellos una prioridad bastante similar. Sin embargo, en la práctica se observan algunas diferencias. Podría pensarse que derivan de discrepancias de orden político-ideológico, pues sc relacionan básicamente con los PSDI y el tratamiento a la inversión extranjera ${ }^{19}$. No obstante, ellas parecen responder más bien a razones de naturaleza puramente económica, vinculadas al nivel de desarrollo y a la estructura productiva de los países. En efecto, Ecuador y Bolivia conceden mayor prioridad a los programas de desarrollo industrial, pues los beneficios que obtendrán con su implementación serán superiores a los que lograrian en ausencia de este mecanismo. Por otra parte, es sintomático que en general tanto los gobiernos como los sectores empresariales de Venezuela y Colom-

\footnotetext{
1: Véase N. Rodriguez, "Venezuela: actores y agentes políticos internos del proceso de integración andina", en CINDA, et al. (cd.), 1974. Alli sc transcribe una seric de acuerdos de FEDECAMARAS, así como las posiciones de los gobiernos de los presidentes Leoni y Caldern entre 1968 y 1973.

${ }^{18}$ En este sentido, la estrategia tradicional deposita menor confianza en la alternativa del incremento y diversificación nacional de exportaciones, sobre todo industriales, orientadas hacia el mercado internacional en general.

${ }^{10}$ También con respecto al PL y al AEC se observan algunas discrepancias, apareciendo Colombia mucho más interesada en acelerar y cxtender la desgravación automática. Esta diferencia con los otros tres países se debe a la mayor diversificación de la cstructura productiva colombiana, esto es, responde a consicleraciones de carácter básicamente económico. Véanse en especial los informes finales de la segunda y tercera reunión del Conscjo de Comercio Extcrior, mimcos, JUNAC, scptiembre y noviembre de 1975, asi como las declaraciones en tal sentido del Representante Titular de Colombia ante la Comisión del Acucrdo, en Revista Decision, № 6, febrero de 1975.
} 
bia hayan respaldado en general las normas de la Decisión $24^{20}$, mientras que los gobiernos y empresarios de Bolivia y Ecuador se mostraban partidarios de hacerlas menos rigurosas. ${ }^{21}$

Cabe señalar, por otra parte, que entre los que siguen la estrategia de desarrollo tradicional figuran los únicos dos países que han mantenido hasta la fecha un sistema político democrático. Círculos colombianos han manifestado que ello los sitúa en una posición relativamente desmedrada, debido a la menor flexibilidad que tiene un gobierno de este tipo para tomar algunas decisiones internas y adoptar o modificar compromisos internacionales, los cuales, por su trascendencia, deben ser discutidos en el Congreso y ante la opinión pública. ${ }^{22}$

Por otra parte, los gobiernos democráticos están más obligados, a diferencia de los otros que predominan en el Grupo Andino, a considerar los planteamientos de sus opositores o de los grupos sociales que no ocupan un lugar dominante dentro del sistema. En este sentido, en las naciones en que impera un régimen democrático es probable que los trabajadores tengan alguna influencia relativamente mayor o que se conceda más prioridad al logro de sus aspiraciones (como un nivel relativamente alto de ocupación, por ejemplo). ${ }^{23}$

${ }^{\circ}$ Un cable de la agencia EFE del 5 de noviembre de 1975 , por ejemplo, reprodujo declaraciones del presidente de la Asociación Nacional de Industrias Financieras de Colombia, en las que este personero advertía que "el poder de las empresas transnacionales es peligroso para el Grupo Andino". Además, expresó preocupación ante la posibilidad de que el Grupo se transformara "cn la carnada" de las voraces transnacionales, que en adelante tendrán un mercado más amplio y atractivo. Terminó dicicndo que en tal evento "éstas y no cl Grupo Andino quedarán más fortalecidas". Seguramente, no todo el sector privado compartc cste criterio; sin cmbargo, similar posición sostuvieron el representante titular de Colombia ante la Comisión del Acucrdo de Cartagena (Diario El Mercurio, 6 de noviembre, 1975, p. 7), y el propio Presidente de la República, durante una visita oficial a Ecuador (Revista Decisión, agosto-septiembre 1975). Cabe scñalar, no obstante, que la actual posición de los empresarios privados de Colombia es resultado de una evolución en los últimos años, puesto que en un comienzo sustentaban una postura diferente.

${ }^{21}$ Ecuador ha sido el país más elocuente en este sentido. Véanse las diversas declaraciones del representante titular ante la Comisión del Acuerdo de Cartagena durante 1975 (Revista Decisión, agosto-septiembre 1975) y del Presidente de la Cámara de Comercio Ecuatoriana. También Moncada, 1975.

${ }^{22}$ Véase editorial del diario liberal El Espectador. Extractado en cable de UPI, del 18 de junio de 1975, en El Mercurio, Santiago, 19 de junio de 1975.

${ }^{23}$ Los grupos asalariados han apoyado la integración fundamentalmente como un medio de aumentar la autonomía nacional de los países (reducir la dependencia externa) y acclerar cl desarrollo económico, planteando, no obstante, la necesidad de adoptar medidas para impedir que los empresarios obtengan beneficios desmesurados en relación a los correspondientes a la sociedad en su conjunto. Véase el acuerdo final del "Encuentro de dirigentes sindicales del Area Andina sobre el proceso de integración económica", organizado por la Central Única de Trabajadores (CUT), diciembre 1970. También Camacho, 1975, y Atria, 
En síntesis, los países que siguen una estrategia de desarrollo de tipo más tradicional tienen un sistema económico mixto, con una apreciación global sobre los problemas del desarrollo relativamente similar a la de los años sesenta. Esto implica, en términos generales, que tal estrategia es relativamente similar a aquella en la cual se basó y en función de la cual se ha estado implementando la integración andina.

\section{La estrategia de libre mercado}

Por el contrario, el pais -Chile- que desde 1973 sigue la estrategia de libre mercado visualiza la integración casi exclusivamente como un proceso tendiente a intensificar su intercambio comercial con los países de la Subregión. Además, se la concibe como una "segunda mejor alternativa" respecto del comercio libre con todo el mundo. En el primer sentido, la dimensión política internacional del Acuerdo -como instrumento de negociación conjunta frente al resto del mundo y fórmula para reducir la dependencia del exterior- tiene menos preponderancia. Por otra parte, esta estrategia considera implícitamente al comercio "libre" con todo el mundo como una alternativa real que permite diversificar exportaciones; en consecuencia, estima que el desarrollo de éstas no debería fundarse en márgenes de preferencia especiales en los mercados de los países miembros.

Esta concepción se traduce en la práctica en un apoyo general al programa de liberación, pero en un marcado rechazo a un AEC que no sea bastante inferior al promedio histórico que han mantenido los países. Por otra parte, tampoco se acepta que el AEC tenga una estructura muy diferenciada por productos; es decir, se postula establecer tasas bajas y con una dispersión muy pequeña ${ }^{24}$. Estas son algunas de sus contradicciones más importantes con el esquema andino de integración.

Las posiciones anteriores emanan de la esencia de la estrategia neoliberal, que consiste en dar preferencia al mercado como mecanismo asignador de recursos, limitando al máximo las políticas gubernamentales que busquen condicionar o alterar algunos de los resultados a que conduciría el "libre" funcionamiento del sistema. En este sentido, desde el punto de vista que hemos llamado políticoideológico implica ciertas reservas frente al uso de los PSDI -vale decir de la planificación industrial - los cuales, como se ha dicho,

et al., "Chile: actores y agentes políticos del proceso de integración andina", en CINDA, et al. (cd.), 1974.

${ }^{24}$ Véanse las observaciones que en este sentido formularon los representantes chilenos en las reuniones del Consejo de Comercio Exterior, JUNAC (1975 d y e) . 
pretenden evitar que la localización de industrias por países quede determinada por el sólo juego del "mercado libre".

Finalmente, también implica una posición ideológica de rechazo a la regulación del capital extranjero, es decir a la Decisión 24. En tal sentido, esta estrategia plantea que el "nacionalismo" constituye una posición obsoleta ${ }^{25}$. La concepción neoliberal, aparte de conceder a la inversión foránea un rol muy activo en la promoción del desarrollo, procura "no discriminar" en su contra, vale decir, trata de otorgarle un tratamiento similar al establecido para la inversión nacional. Esta actitud ha desencadenado - por su clara discrepancia con la que anima al Acuerdo de Cartagena- un serio conflicto, que es suficientemente conocido. ${ }^{26}$

Desde el punto de vista de los grupos sociales que apoyan esta estrategia, el más importante es el sector privado industrial moderno, junto con una tecnocracia que actúa desde puestos del gobierno, formada en una escuela de pensamiento político económico favorable al libre mercado. ${ }^{27}$

\section{La estrategia de desarrollo planificado}

Esta es la estrategia que sigue el Perú, caracterizada, en general, por el rol activo que asume el Estado en ella, muchas veces en franca contradicción con el sector privado ${ }^{28}$. Y el mayor énfasis concedido a la dimensión política internacional de la integración como vehículo para obtener un desarrollo nacional autónomo. ${ }^{29}$

Dicha estrategia se funda en una activa planificación estatal, antes que en la "libre" operación de las fuerzas de mercado. En consecuen-

${ }^{25}$ Véase Harry Johnson (ed.), Economic nationalism in old and new States.

20Para un recuento de las discusiones en la Comisión del Acuerdo de Cartagena sobre este tema en 1974, véase Grupo Andino (Carta informativa oficial de la JUNAC), No 41, noviembre 1974; las principales versiones periodísticas cstán en las revistas Informador Andino y Decisión de 1974.

${ }^{27}$ Véase reportaje de la revista Qué Pasa, de Santiago, No 214, sobre el origen profesional de las principales autoridades económicas civiles del actual gobicrno chileno.

${ }^{28}$ Véase Bernales, "Perú: actores y agentes políticos internos de integración andina", en CINDA, et al. (ed.), 1974, y declaraciones públicas de inclustriales, como el presidente de la Asociación de Exportadores del Perú (ADEX), según las cuales la falta de confianza de los empresarios conspira contra la realización de las inversiones necesarias para aprovechar el mercado ampliado. Revista Decisión, $\mathrm{N}^{\circ}$ 6, febrero de 1975, p. 31.

${ }^{20}$ Ver, por ejemplo, discurso del Presidente Juan Velasco Alvarado con motivo de la inauguración de la sede de JUNAC en Lima. Extractos en Iñiguez y VegaCenteno (1975). 
cia, otorga menor importancia al PL y una más elevada a los PSDI ${ }^{30}$. Procura además que se establezca un AEC promedio alto y más diferenciado para desarrollar industrias estratégicas ${ }^{31}$. Cabe señalar asimismo que el modelo de desarrollo peruano procura una participación considerable de empresas de propiedad social en sectores estratégicos de la economía, muchos de los cuales deberán ser objeto de PSDI. ${ }^{32}$.

Esta estrategia nacional asigna un rol restringido y regulado al capital extranjero e involucra un apoyo categórico a la Decisión 24, a la que se otorga, por otra parte, tanta o más prioridad como a los demás programas y mecanismos de integración. Las declaraciones de altos personeros del gobierno son muy explicitas al respecto.

"Nosotros creemos que Decisión 24 es la mejor garantía para que el desarrollo de nuestros pueblos sea un desarrollo autónomo que vea, antes que nada, los intereses de nuestros países. Su cumplimiento debe ser tan estricto como las desgravaciones, porque creemos que si no se respetan plenamente todos los acuerdos, entonces no hay razón para respetar solamente algunos, aquellos que pueden convenir a tal o cual país". ${ }^{33}$

${ }^{30}$ Asi, por ejemplo, en mayo de 1975 el representante peruano ante la Comisión del Acuerdo de Cartagena declaró que desde el inicio del proceso de integración subregional su pais sostuvo una tesis muy clara: "que cl esquema de integración a aprobarse debcría constituir un instrumento al scrvicio del desarrollo cconómico de los países miembros, vale decir, que debería incidir básicamente en el crecimiento industrial de los mismos. Por eso, hizo énfasis en el mecanismo de programación industrial como el más apropiado para lograr el desarrollo equilibrado y armónico de la Subregión, no dejando liberado el proceso a la simple liberación arancelaria". Véase El Informador Andino, año 4, No 42, junio 1975 , p. 1 .

${ }^{a}$ Ver posición de representantes del Perú en las reuniones del Consejo de Comercio Exterior, op. cit. Para un breve resumen de las diversas formas de propiedad de empresas que consulta cl modclo peruano, véanse Iñigucz y VegaCenteno (1975).

s2Las condiciones de operación de empresas estatales que no se rigen por criterios de mercado, ha sido causa de constantes polćmicas dentro del Grupo Andino. Colombia mostró una particular sensibilidad por este aspecto durante el periodo 1971-73 debido a la forma en que cstaba siendo modificado el sistema económico chileno. Véasc Rosthlisberger, D. "Colombia: actores y agentes políticos internos del proceso de intcgración andina", en CINDA, et al. (ed.), 1974, y también Ffrench-Davis (1973).

${ }^{53}$ Alberto Jiménez de Lucio, Ministro de Industria y Turismo del Perú. Entrevista aparecida en el diario El Correo de Lima, reproducida en El Informador Andino, Ne 42, julio de 1975. 
III. - POSICIÓN DE LOS PAÍSES ANTE LOS EFECTOS ECONÓMICOS DEL PROCESO

En esta sección el análisis se concentrará en los efectos más puramente económicos para cada país que se derivan de la aplicación de los principales instrumentos consultados en el proceso de integración subregional. En cierto sentido, identificar y comparar estos cfectos es más fácil que en el caso de los político-ideológicos. Aquéllos se miden cn relación a un parámetro homogéneo, como es el aporte de cada mecanismo al Producto Nacional del país de que se trate, el cual exhibe una determinada estructura económica, mientras que los segundos deben ser referidos al concepto más difuso de la consolidación de detcrminadas estrategias de desarrollo y se relacionan también con las posicioncs político-ideológicas de los gobiernos. Además, para el estudio de los electos económicos existe cierto método o teoría ya desarrollada.

No obstante, el análisis y la cuantificación de ellos no están exentos de dificultades, como son el hecho de que los supuestos de la teoria tradicional son a menudo irreales o restrictivos y, sobre todo, que la integración y el desarrollo constituyen procesos dinámicos, por lo que toda estimación acerca de la incidencia futura de determinado instrumento envuelve algunas dosis de incertidumbrc ${ }^{34}$. No obstante, aquí se hace un esfuerzo preliminar por estimar cualitativamente los efectos económicos generales de mediano plazo (piénsese en 1 a 5 años) que se derivan de la estructura económica actual de los paises, ${ }^{35}$

Desde el punto de vista económico, la fuente esencial de beneficios de la integración para un país es la posibilidad de acclerar, a través de la especialización, el crecimiento del producto nacional, exportando cicrtos biencs (especialmente manufacturados) hacia el mercado común. Ello se logra esencialmente en virtud de la liberación de restricciones y gravámenes al comercio entre los países que se integran, junto con la imposición de un arancel externo común frente a ter-

\footnotetext{
"Además, detcrminada variable puede tener un efecto negativo en el corto plazo, pero positivo en el más largo, y viceversa. Incluso, incurrir en ciertos "costos" inmediatos puede resultar ineludible para conseguir otros beneficios en el futuro.

${ }^{35}$ Nótese entonces que el tema tratado en este capitulo se refiere implícitamente tanto al difícil problema de los efectos económicos de la integración, como al de la distribución de beneficios entre las naciones participantes en el proceso.
} 
ceros; esto es, gracias al establecimiento de cierto margen de preferencia para la producción regional.

Lo anterior implica -en otras palabras- que el pais recibe, como exportador, un precio más alto que el internacional por los bienes vendidos en la Subregión. A cambio de los beneficios "brutos" obtenidos por ese concepto, el país considerado está dispuesto a incurrir eventualmente en cierto "costo", al aceptar importar ciertos bienes desde el mercado común a un precio también más alto que el que ellos tienen en el resto del mundo, determinado justamente por ese margen de protección a la producción regional. Sin embargo, como este intercambio recíproco implica que: i) cada país se especializa en lo que produce a menor costo dentro del mercado común, y ii) se aprovechan economías de escala e innovaciones tecnológicas, gracias al tamaño mayor del mercado, siempre existen condiciones para que los beneficios sobrepasen los costos para cada país en particular y, eventualmente, para todos ellos en forma simultánea.

La teoría más tradicional de la integración ${ }^{36}$ postula que los beneficios del proceso corresponden únicamente al bien conocido efecto "creación de comercio", que surge cuando, gracias a la liberación del intercambio intrarregional, un país comienza a importar bienes que hasta entonces había estado produciendo a un precio interno más alto. Los "costos" de la integración se asocian tradicionalmentc a la "desviación de comercio", que se deriva del pago de precios superiores a los internacionales por importaciones desde los demás integrantes de la Subregión. En consecuencia, los beneficios netos para un país particular resultarian mayores cuanto más significativo sea el efecto creación de comercio, comparado con su desviación.

Dicha teoría adolece no obstante, de varias limitaciones. Por una parte, el planteamiento de Viner se refiere sólo a los efectos de los cambios en la producción, con precios de los bienes tales que no alteran el consumo. El trabajo pionero de Lipsey (1960) consideró también el efecto consumo o de sustitución entre bienes debidos a los cambios de precios relativos, lo cual implica costos o beneficios adicionales. ${ }^{37}$

Por otra parte, esta teoría supone además competencia perfecta y un grado mínimo de estabilidad en todos los mercados, pleno empleo de los recursos de capital y de la fuerza de trabajo, alta movilidad de los factores productivos y otras situaciones particularmente poco realistas en países subdesarrollados, aparte, de limitarse a los efectos estáticos de la integración. ${ }^{38}$

${ }^{30} \mathrm{~J}$. Viner, "The economics of custom union", en P. Robson (ed.). 1972.

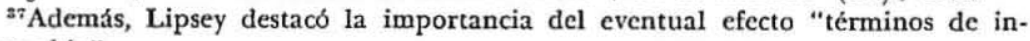
tercambio".

${ }^{38}$ Mikcsell, R., "The theory of common market and developing countries", en Robson (ed.), 1972. 
En relación a este último aspecto, numerosos estudios han destacado la importancia de los efectos dinámicos o de más largo plazo que el proceso acarrea, entre los cuales se cuentan la generación de mayor competencia, el aprovechamiento de economías externas y de escala ${ }^{30}$, y el aprendizaje de nuevas técnicas productivas y de comercialización externa que pueden llevar a reducir los costos hasta niveles que hagan posible incluso desarrollar nuevas exportaciones hacia el resto del mundo. Por último, cabría agregar los efectos del aprovechamiento de la capacidad instalada no utilizada en mercados nacionales reducidos, así como el mayor empleo que por esta vía puede generar la integración.

Sin desconocer la importancia eventual de estos efectos dinámicos, en la presente sección se prestará más atención a los efectos estáticos de los distintos programas de integración sobre cada país, pero a partir de las características más bien estructurales o permanentes su economía. Entre ellas se pondrá especial énfasis en el nivel de desarrollo y de industrialización, así como en el volumen y estructura de la producción interna y del comercio exterior. Esto obedece no sólo a la dificultad de evaluar simultáneamente la magnitud de varios efectos dinámicos, sino debido también a que de hecho los países reaccionan a menudo más en función de problemas de corto o mediano plazo.

Antes de entrar a analizar los efectos generales más probables de cada uno de los instrumentos, -vale decir, del PL, el AEC, los PSDI y el régimen común frente al capital extranjero- cabe destacar que como todo fenómeno económico, los programas de integración afectan en forma diferenciada a los distintos grupos sociales que coexisten dentro de cada país. Procuraremos hacer explícitos estos efectos, en los casos en que ellos resulten más inequívocos, sobre tres tipos de agentes económicos.

En primer lugar, el Estado es uno de los agentes sociales que se ve más directamente afectado con un proceso integracionista. Sus ingresos tributarios pueden aumentar o reducirse, en función del volumen relativo del comercio del país y de la relación entre los aranceles iniciales y el AEC definitivo. También habría que considerar aquí los menores recursos que el Estado debe gastar en promover exportaciones no tradicionales dentro de un mercado común, comparados con los que tendría que utilizar para obtener los mismos niveles de exportaciones en mercados de terceros países. Por otra parte, el Estado puede verse afectado a través del cambio en los excedentes (o pérdidas) de las empresas públicas, especialmente

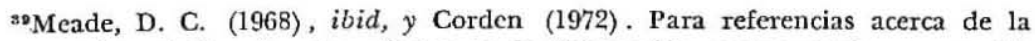
importancia práctica de estos efectos en la integración centroamericana, ver Lizano (1975). 
de las que compiten con importaciones o exportan hacia el mercado común.

El segundo grupo social o agente económico que se ve muy directamente afectado está constituido por los empresarios privados. Aquí cabe distinguir dos sectores: los exportadores hacia la Subregión, que captan el grueso de los beneficios que genera el proceso, y los productores de bienes que deben competir con similares que, en virtud de la liberación, comienzan a ser importados desde el resto del área ${ }^{40}$. Dado el sistema económico vigente en la mayoría de los países, son los integrantes de este grupo los que mayor influencia tienden a ejercer en las decisiones, y a menudo pareciera que son los únicos afectados por la integración.

Por tal razón, la disputa entre esos dos sectores empresariales en cada país estaría determinando básicamente su adhesión o rechazo al proceso. Normalmente, en esa disputa participan no sólo firmas privadas nacionales, sino también estatales $y$, sobre todo, corporaciones extranjeras que tienen establecidas o están instalando filiales en cada país. Estas últimas plantean la dificultad adicional de comportarse no de acuerdo a un criterio de maximización de sus propias utilidades, sino en función de los intereses de la corporación en su conjunto. ${ }^{41}$

Un tercer grupo social o agente económico real o potencialmente afectado por la formación de un mercado común es el constituido por los trabajadores, en general, y por los ocupados en las empresas más directamente vinculadas al proceso, en particular. Ello se verán favorecidos por la integración en la medida que induzca un aumento de los salarios reales, y permita generar más empleo e incrementar la participación de los asalariados en el ingreso nacional y en las decisiones económicas. Lo primero dependerá de la intensidad con que la producción sea orientada hacia la elaboración de bienes relativamente más intensivos en el empleo de mano de obra; y en este contexto, el AEC, en particular, juega un rol determinante. Lo segundo podría alcanzarse especialmente a través de la participación de los trabajadores, del Estado y de otros sectores sociales en la propiedad de las nuevas industrias que se desarrollen gracias a la integración. ${ }^{42}$

Por último, cabe agregar que los grupos anteriores, así como otros distintos, también son afectados por un proceso de integración en su

${ }^{40}$ Los importadorcs serían otro de los grupos afectados, pero no siempre cons. tituyen uno distinto; a menudo son los propios productores los que importan desde el exterior.

"Para un análisis más detallado de las implicaciones de este comportamiento, véase Tironi (1975).

${ }^{12}$ Perú ha aprovechado esos programas para la consecución de tal objetivo. Véanse Iñiguez y Vega-Centeno, cit. 
calidad de consumidores de bienes cuyos precios relativos cambian. Los consumidores pueden ser empresas, en el caso de productos intermedios, o individuos y familias, en lo que se refiere a bienes finales. En general, se verán favorecidos los consumidores de bienes cuyos aranceles externos comunes resulten inferiores al nivel imperante inicialmente en el país, o cuyos precios bajen gracias a la mayor eficiencia de la producción subregional, y viceversa. Los consumidores se verán afectados no solamente por los cambios de los precios que pagan por los productos transados dentro del mercado común, sino también por las fluctuaciones de aquellos bienes que sigan adquiriendo en el resto del mundo, ya que el AEC afecta a todo el universo de productos. Por último, no debe olvidarse que los consumidores no son homogéneos; las familias y los individuos tienen distintas estructuras de consumo, las cuales dependen de la distribución del ingreso imperante en el país ${ }^{43}$. En general, es probable que los productos manufacturados reduzcan sus precios relativos en comparación con los de los bienes agropecuarios, lo cual, ceteris paribus favorecería relativamente más, en cuanto a consumidores, a los grupos de ingresos medios y altos, porque en su patrón de consumo el primer tipo de productos alcanza una ponderación muy elevada.

De estos cuatro grupos o agentes sociales, los que normalmente han tenido menos capacidad de hacer valer sus intereses son los dos últimos: los consumidores y los trabajadores. Esto explicaría la relitiva indiferencia que han mostrado frente al proceso, lo cual no significa que no resulten o no puedan verse afectados por él ${ }^{44}$. Por eso, es importante destacar que el grado de indiferencia, adhesión o rechazo global al proceso de integración dependerá también del poder que cada uno de estos grupos tenga en las decisiones relevantes, lo cual, a su vez, aparcce reflejado principalmente en la cstrategia global de desarrollo que sigue el país de que se trate.

\section{Efectos del programa de liberación}

El proceso de liberación es el que se relaciona más directamente con la forma como se ha concebido la integración en la literatura teórica (neoclásica) tradicional, ya que corresponde a la parte del comercio

\footnotetext{
"sara una discusión sobre algunas relaciones entre integración y distribución del ingreso en los países de la subregión andina, véasc Figueroa (1975).

"Además, el hecho de que la integración en si misma afecte de determinada manera a los distintos grupos sociales no implica que tales consecuencias sean ineludibles ni tampoco recomendables. Siempre será posible recurrir a mecanismos o politicas redistributivas internas capaces de compensar los efectos no deseados que recaen sobre ciertos grupos sociales.
} 
que se desarrollaría gracias a la operación del mercado. En este sentido, se cuenta con un instrumental más sofisticado para analizar el problema, pero no por ello más útil, debido al escaso realismo de muchos supuestos y a la dificultad de incorporar efectos dinámicos ${ }^{45}$. Para aislar los efectos que este programa genera per se es necesario suponer por el momento que el arancel imperante en un principio en cada país resulta igual al AEC que se fija para todo el grupo ${ }^{46}$, y concentrarse en el comercio generado a través de la iniciativa de las unidades productivas que actúan autónomamente en el mercado.

En ese contexto, conviene distinguir entre el efecto producción y el efecto precios de un proceso de integración. En lo concerniente a los efectos de producción solamente -es decir, dados ciertos precios a ciertos márgenes de protección subregional- el programa de liberación per se será más favorable para aquellos países que produzcan un mayor volumen de bienes susceptibles de ser exportados al resto del área. Por el lado del efecto precios, dado un incremento equis de las exportaciones, los beneficios para un país serán mayores cuanto más alta sea la protección regional que se le conceda, es decir, cuanto más alto sea el AEC sobre esos bienes comparado con el que rija para los productos que debe importar desde el resto del área.

Extendiendo el análisis del efecto precios, cabe agregar que el programa de liberación será más favorable para aquellos países que gracias a la ampliación del mercado logren reducir el costo de producción de sus exportaciones. Esto ocurrirá especialmente con respecto a bienes manufacturados que presentan economías de escala o se producen en plantas que no podían utilizarse a plena capacidad antes de la integración. Los beneficios se traducen tanto en mayores excedentes para los exportadores, como en menores precios domésticos para los consumidores de ese país y de sus asociados (importadores).

Con respecto a las importaciones, el PL debería favorecer relativamente más a los países que debido a las características de sus estructuras de producción y de consumo importen desde la Subregión bienes cuyo margen de protección (AEC) sea bajo. EI precio final de éstos sería así relativamente similar al que los consumidores de tales países pagarían en caso de adquirirlos fuera del área.

Por lo tanto, dados ciertos márgenes de protección regional, los beneficios tenderán de ser mayores para aquellos países que, por un lado, puedan expandir más su oferta de productos exportables al

\footnotetext{
45V́ase especialmentc Lipsey (1960), Meade (1968) y Johnson (1958) en Robson (ed.), op. cit.

${ }^{*}$ Se trata, como es obvio, de un supuesto operacional, que no podrá cumplirse en forma simultánea para todos los países a menos que en todos ellos los aranceles iniciales hayan sido semejantes.
} 
mercado común y, por otro, tengan una mayor proporción de su demanda abastecida con producción doméstica comparada con importaciones desde fuera del área integrada.

Finalmente, cabe señalar que la parte más visible de los beneficios de un programa de liberación comercial se refleja de manera directa en los excedentes captados por los exportadores. Otra parte -más difícil de estimar-se traduce en última instancia en precios más bajos para los consumidores, que se explican por la especialización y el aprovechamiento de las economías de escala. Estos efectos incluyen en forma implícita las ventajas derivadas de la mejor asignación de recursos que se logra cuando el país deja de producir internamente bienes que puede comprar más baratos en el resto de la Subregión. Por el lado de los costos en que el PL per se obliga a incurrir, estos tienden a reflejarse principalmente en un descenso de la recaudación por concepto de ingresos arancelarios.

En la medida en que la experiencia relativamente reciente pueda utilizarse para hacer una estimación de los efectos futuros más probables de cada programa -aunque sólo sean los estáticos y de mediano plazo-, la aplicación del criterio anterior sugiere que el PL como tal, es decir en cuanto a mecanismo de integración, resultaría en general más ventajoso para Colombia y Chile, y menos favorable para Venezuela, Bolivia, Ecuador y Perú ${ }^{47}$. Ello se derivaría de las siguientes consideraciones gencrales:

i) Por el lado de las oportunidades de exportar, el PL afecta principalmente a los bienes manufacturados que tenían los más altos aranceles antes de la integración. En ese sentido, favorece más a los países que exhiben un mayor grado de industrialización, que ya producían internamente esos bienes ${ }^{48}$. La experiencia y el conocimiento adquirido en la etapa de sustitución nacional de importaciones, unidos a las capacidades instaladas no utilizadas y a las economías de escala, así como las externalidades y la existencia de una infraestructura más desarrollada son factores que permiten aprovechar mejor tales posibilidades. Es difícil, sin embargo, cuantificar estos aspectos, y las cifras históricas no siempre son relevantes. ${ }^{40}$

\footnotetext{
4No obstante, en la forma concreta que ha asumido el PL dentro del proceso de integración subregional se contemplan modalidades para compensar las desventajas que pudieran recaer por este concepto sobre las naciones de menor desarrollo relativo del área. Véase al respecto A. Aninat (1975).

${ }^{48}$ Véase Caballero (1975) para un análisis del caso colombiano.

${ }^{40}$ No obstante, en el Cuadro $l$ se presentan algunas cifras indicativas agregadas de carácter preliminar. Las columnas (5) y (6) muestran que Colombia dirige una fracción relativamente alta y creciente de sus exportaciones hacia el mercado subregional. En cl otro extremo, Venezuela y Perú colocan una fracción pequeña, aunque también en ascenso, debiendo considerarse además que el pri-
} 
ii) Por el lado de las importaciones totales, Colombia ha tenido el menor porcentaje de su oferta agregada abastecida desde el exterior (alrededor del $7 \%$ en 1972), mientras que Bolivia y Venezuela han tenido el 14 y el $18 \%$ respectivamente. Ello implica que sustituir esas importaciones que se adquieren más baratas en el resto del mundo, por otras más caras provenientes del mercado común, tiene para Bolivia y Venezucla un costo proporcionalmente más elevado. ${ }^{50}$

\section{Cundro 1}

SIGNIFICACIÓN DEL COMERCIO EXTERIOR TOTAL Y SUBREGIONAL EN LOS PAf́SES ANDINOS: 1972 Y 1974

\begin{tabular}{|c|c|c|c|c|c|c|}
\hline \multirow[t]{2}{*}{ Paíscs } & \multirow{2}{*}{$\begin{array}{c}\text { PGB } 1972 \\
\text { (milloncs } \\
\text { de dólares) } \\
\text { (1) }\end{array}$} & \multirow{2}{*}{$\begin{array}{l}\text { Coeficiente } \\
\text { de abaste- } \\
\text { cimiento } \\
\text { externo a/ } \\
\text { (2) }\end{array}$} & \multicolumn{2}{|c|}{$\begin{array}{c}\text { Cocficiente } \\
\text { de abaste- } \\
\text { cimiento } \\
\text { subregio- } \\
\text { nal b/ }\end{array}$} & \multicolumn{2}{|c|}{$\begin{array}{l}\text { Coeficiente } \\
\text { de exporta- } \\
\text { ciones sub- } \\
\text { regionales }\end{array}$} \\
\hline & & & $\begin{array}{l}1972 \\
(3)\end{array}$ & $\begin{array}{l}1974 \\
(4)\end{array}$ & $\begin{array}{l}1972 \\
(5)\end{array}$ & $\begin{array}{c}1974 \\
(6)\end{array}$ \\
\hline Bolivia & 1.366 & 14,4 & 2,8 & 5,0 & 8,8 & 8,3 \\
\hline Colombia & 12.261 & 6,7 & 3,7 & 6,1 & 9,3 & 13,5 \\
\hline Chile & 8.821 & 19,7 & 4,5 & $12,9 \mathrm{~b} /$ & 2,4 & 2,7 \\
\hline Ecuador & 3.052 & 12,0 & 10,7 & $20,5 \mathrm{~b} /$ & 8,6 & 16,4 \\
\hline Perú & 7.665 & 11,0 & 7,5 & $16,1 \mathrm{~b} /$ & 3,4 & 4,5 \\
\hline Venezuela & 12.957 & 18,1 & 0,8 & 1,9 & 1,3 & 1,8 \\
\hline
\end{tabular}

a/ Importaciones totales como porcentaje del PGB en 1972.

b/ Importaciones de la Subregión como porcentaje de las importaciones totales. Cabe resaltar que las de Chile, Ecuador y Perú resultan muy afectadas por la inclusión de Venezucla, debido principalmente a que ellos son importadores netos de petróleo a un precio que en la actualidad es muy superior al de 1972. Excluyendo el comercio con Venezucla, el cocficiente de abastecimiento subregional cae de 12,9 a 9,7 para Chile, de 20,5 a 7,9 para Ecuador, y de 16,1 a 9,5 para cl Perú.

Fuente: Columnas (1) y (2), Ffrench-Davis, 1975, Cuadro 1; el resto, JUNAC, "Grupo Andino: comercio exterior global en 1965-74, mimco, Cuadro 13; 25-8-75.

mero se incorporó al PL recién en 1973. Ecuador y Chile constituyen casos especiales, por lo que las cifras pueden inducir a conclusiones erróneas. El primero aparece con coeficiente de exportaciones subregionales muy alto, debido al aumento del precio del petróleo. En el caso del segundo, la cifra resulta inferior a la que cabría esperar en el largo plazo, debido a que en 1974 el precio del cobre fue excepcionalmente alto; esto incrementa el valor de las exportaciones totales (el denominador del cocficiente), sin hacer lo mismo con el numerador porque Chile vende muy poco cobre en el mercado andino. Por último, hay que tener en cuenta que este pafs ha vivido etapas de gran inestabilidad en los últimos años, que repercutieron negativamente sobre sus exportaciones a la Subregión.

${ }^{60}$ Hasta 1974 ese desvio habría sido para Venezuela, Colombia y Bolivia menos significativo que el que afecta a Ecuador, Perú y Chile. En el caso de este último 
Tan importante como los aumentos globales de exportaciones e importaciones resulta la composición de ellas. Ya se destacó que en lo concerniente a las primeras, las naciones más desarrolladas están, por concepto del PL, en mejores condiciones de exportar manufacturas, mientras que los demás países tenderán a seguir concentrándose -al menos en el corto plazo- en bienes de menor grado de elaboración ${ }^{51}$. Por el lado de las importaciones desviadas en terceros países, en las naciones menos industrializadas (Bolivia y Ecuador) ellas afectarían principalmente a los bienes de consumo ya producidos y -de establecerse un margen de protección razonable- susceptibles de ser exportados por Colombia, Chile, Venezuela y, en algún grado, Perú. En cambio, la desviación por parte de estos últimos -en ausencia de otros programas compensatorios, como los PSDI-, serían importados desde el resto del mundo a un costo más bajo.

En términos de los agentes socioeconómicos afectados por el PL, una parte significativa de los costos provenientes de la desviación del comercio (en particular, respecto de los bienes que no eran producidos inicialmente en los países y que ahora se importarían desde la región) pueden recaer sobre el Estado ${ }^{52}$. Dichos costos se pueden medir en función de los menores ingresos recaudados por concepto de aranceles. ${ }^{53}$

Desde el punto de vista del Estado, lo relevante es observar la significación de esos menores ingresos arancelarios en relación con i) la importancia que el conjunto de ellos tiene dentro del total de la recaudación fiscal, y ii) con la significación de las importaciones de origen regional dentro del total de las compras en el exterior ${ }^{54}$.

país, también las cifras de 1974 sobrestiman los posibles efectos desfavorables del PL, debido a las importaciones de petróleo. Los datos de 1972 pueden ser más representativos al respecto.

${ }^{61}$ Este fenómeno se ha suscitado con alguna intensidad en Bolivia $y$, en menor grado, en Ecuador, pese a que el PL andino consulta una liberación más rápida de los mercados de los demás países del área, los cuales pueden ser abastecidos desde estas dos naciones.

${ }^{52}$ Naturalmente cuando el Estado restringe importaciones por medio de cuotas o prohibicioncs la desviación de comercio no se traduce en menores ingresos estatales sino en menores rentas de los importadores privilegiados por la detención de las cuotas.

${ }^{83}$ Estos no representan el efecto total de la integración sobre los ingresos estatales. Normalmente se suscitan también beneficios (o costos adicionales) derivados de los ajustes en el AEC.

${ }^{5}$ En términos más rigurosos, el efecto mencionado depende del grado en que esas importaciones subregionales sustituyen verdaderamente importaciones procedentes de terceros países y no producción nacional. Sin embargo, se carece de estadísticas sobre este aspecto en particular. Los resultados que se obtengan de comparar el conjunto de las importaciones subregionales con el total constituirán pues una primera aproximación. 
Las cifras pertinentes para cada país se reúnen en el Cuadro 2. En este sentido, cabe señalar que Ecuador, que parecería relativamente más afectado por el PL, es también uno de los países en que el Estado depende más de aquella fuente de recursos, y por lo tanto, estaría haciendo el mayor sacrificio por este concepto. Lo seguirían Bolivia y Perú. Este último se encuentra en tal situación pese a que ocupa el 49 lugar en relación a la importancia de los ingresos arancelarios (con sólo el $19 \%$, comparado con el $32 \%$ de Bolivia), debido a que su coeficiente de abastecimiento subregional era casi tres veces superior al de este último. En el otro extremo, Venezuela se vería mucho menos afectada, quedando Colombia y Chile en una situación intermedia.

\section{Cundro 2}

SIGNIFICACIÓN DE LA RECAUDACIÓN ARANCEI.ARIA EN LOS INGRESOS FISCALES: 1972

\begin{tabular}{lc}
\hline Paises & Significación (\%) \\
\hline Bolivia & 36,8 \\
Colombia & 18,1 \\
Chile & 10,5 \\
Ecuador & 31,6 \\
Perú & 19,3 \\
Venezuela & 7,8 \\
\hline
\end{tabular}

Fuente: Informaciones Programa Finanzas Públicas OEA. Citado por Aninat (1975), Cuadro 21.

Desde el punto de vista de los efectos sobre los empresarios, la significación que alcanzan las importaciones subregionales dentro del total de éstas en cada país da una idea del impacto de la integración sobre los productores de sustitutos de importaciones ${ }^{55}$. En este sentido, de mantenerse la situación que se apreciaba en 1972, los más reticentes, una vez más, serían los empresarios ecuatorianos, seguidos por los peruanos y chilenos y, en última instancia, por los venezolanos y bolivianos.

En cambio, el apoyo de los grupos exportadores sería más marcado en Colombia y, menor, por el momento, en Bolivia y Ecuador. En Chile y Venezuela era previsible que en un comienzo asumieran una actitud relativamente indiferente o expectante. Sin embargo, los cam-

\footnotetext{
${ }^{6}$ En rigor, un indicador más adecuado lo constituiría la significación de esas importaciones dentro de la producción local de los mismos bienes, y no del total de importaciones. No se dispone, empero, de esa información.
} 
bios ocurridos en el primer país en 1974 y 1975, relacionados con las alzas del tipo de cambio y la profunda depresión de los mercados internos, deberían conducir a una modificación radical de la posición de esos productores, transformándolos en decididos defensores de la integración dentro del país. ${ }^{56}$

En lo tocante a Venezuela, es sabido que recién a fines de 1973 se incorpora al Acuerdo de Cartagena, y que su ingreso a la Subregión obedece en gran parte al cambio de actitud de los empresarios privados. No obstante, el PL en sí mismo difícilmente va a ser el mecanismo del Acuerdo que les reporte los mayores beneficios, debido a las características estructurales de la economía venezolana. En efecto, la altísima productividad del petróleo determina que sea relativamente menos conveniente la producción local o las exportaciones de manufacturas local comparada con las importaciones desde el resto del mundo. Por el lado de los costos, esta situación implica niveles salariales más elevados que los vigentes en los demás países del área; por el lado de la demanda, se expresa en que la tasa de cambio de equilibrio (bolivares por dólar) resulta demasiado baja para hacer rentables las posibles exportaciones de manufacturas. Por eso el país les otorga importantes subsidios y tiene mayor interés relativo en los PSDI y, en particular, en aquellos que son intensivos en capital, porque su ventaja comparativa se encuentra en la abundancia de este recurso.

Cabría insistir, para terminar, que el PL asume distintas modalidades -excepciones, diferentes velocidades de desgravación, etc.destinadas fundamentalmente a atenuar los costos de la transición y a favorecer a los países de menor desarrollo relativo. El espacio no permite analizarlas aquí, pero cabe señalar al menos que el efecto de tales modalidades no ha sido insignificante: gracias a ellas, Ecuador, sobre todo, ha aumentado considerablemente sus exportaciones de manufacturas a la Subregión.

En sintesis si hubiera que hacer un ranking de los paises en función de los efectos económicos más favorables que obtendrían de la aplicación del PL solamente, y a partir de sus características estructurales actuales, probablemente Colombia se ubicaría en el primer lugar, Chile debería acercarse rápidamente a una situación parecida; para Bolivia y Ecuador, el PL en sí mismo tendría efectos menos favorables, en tanto que Perú con Venezuela se ubicarían en una posición intermedia.

${ }^{50} \mathrm{En} 1974$, las cxportaciones chilenas hacia el mercado andino aumentaron en 250 por ciento respecto de 1972 . Cifras preliminares indican que los embarques hacia cse mercado alcanzaron durante 1975 un nivel bastante más alto que el registrado en el año precedente; véase Banco Central de Chile, "Intercambio comercial con ALALC", mimco, junio de 1975. 
La segunda condición para incorporarse a los beneficios del mercado común es que cada país mueva sus aranceles nacionales hacia un AEC. Esto ya se comenzó a hacer en 1971, al ser ajustados éstos a un Arancel Externo Mínimo Común (AEMC). La más importante característica específica de este mecanismo es que afectará los precios de muchos bienes que, a pesar de la formación del mercado común, deberán seguir siendo importados desde terceros países. Este aspecto se considerará separadamente, al igual que el cambio del arancel promedio sobre todos los bienes y la estructura de éste.

El nivel promedio del AEC determina el incentivo general que se otorga a la producción destinada a la Subregión y al consumo interno; indirectamente, determina también el estímulo que se concede a las exportaciones dirigidas al resto del mundo. Un AEC más bajo involucra un mayor incentivo a lo segundo; en consecuencia, resultaría más ventajoso para los países que están en mejores condiciones relativas de colocar en el resto del mundo bienes que, gracias a la integración, pueden producir a menores costos. Este sería el caso de Colombia en particular, dado que se encuentra más cerca de terceros países con mercados importantes, como son Centroamérica, el Caribe y Estados Unidos.

Tanto o más relevante que las modificaciones en el nivel promedio de los aranceles, son las que afectan su estructura, es decir, las que alteran la protección relativa de que gozan los distintos tipos de bienes. Naturalmente, esos cambios dependen de la diferencia entre el AEC y el arancel nacional que tenía cada bien antes de la integración.

La estructura arancelaria en cada país y el AEMC imperantes en diciembre de 1973 -considerando una estimación de las exenciones habituales- se presenta en el Cuadro $3^{57}$. Además, allí se presenta, respecto de los aranceles nominales vigentes, una desagregación entre bienes de consumo, intermedios y de capital. Estas últimas cifras no son estrictamente comparables con las anteriores, pero dan una idea de las diferenciales entre tipos de productos.

\footnotetext{
${ }^{57}$ En toda esta sección se supone que el AEC que proponga la Junta será un común denominador entre los aranceles nacionales, aunque algo más bajo que el promedio de ellos. Por eso se toma el AEMC como una referencia aproximada. Hasta el momento de escribirse este articulo la Junta no ha divulgado los aranceles comunes específicos que propone, o un promedio de ellos, sino que rangos por grupos de productos.
} 


\section{Cuadro 3}

PROMEDIOS ARITAÉTICOS DE ARANCELES NACIONALES Y DE AFMC POR THYO DE BIENES AL 31-XII-73

(porcentajes)

\begin{tabular}{lccccccc}
\hline Bicnes de & Bolivia & Colombia & Chile & Ecuador & Perú & Venezucla & AEMC \\
\hline Consumo & 31 & 93 & 190 & 145 & 137 & n.d. & 54 \\
Insumos & 41 & 38 & 118 & 73 & 69 & n.d. & 36 \\
Capital & 31 & 39 & 95 & 49 & 48 & n.d. & 39 \\
Promedio a/ & 19 & 46 & 78 & 21 & 69 b/ & 36 & 41 \\
\hline
\end{tabular}

a/ Este promedio no es comparable con los aranceles desagregados para las tres categorias de productos (línea 1 a 3 ) porque se reficre a aranceles netos de las exenciones que normalmente se aplican en cada pais.

b/ Corresponde al promedio de aranceles nominales, porque no se contaba con información sobre exenciones. Por lo tanto, no es comparable con el de los demás y sobrestima el arancel promedio verdadero.

Fuente: Documentos de trabajo JUNAC citada por Aninat (1975), Cuadros 17 y 18 y "El nivel del Arancel Externo Común", JUNAC, CCE/II/dt 12, mayo 1975 .

Los países de menor desarrollo relativo y Venezuela deberían subir su arancel promedio para alcanzar el AEC probable; el caso más notable lo constituiría Bolivia, que necesitaría duplicarlo. En cambio, los demás países deberían reducirlo; más acentuadamente Chile (a casi la mitad de los que efectivamente cobraba en 1973) y algo menos Perú (en alrededor de un tercio como promedio) ; en cuanto a Colombia, sólo debería hacer un ajuste pequeño.

En cuanto a la estructura del AEC, lo más probable es que el arancel que se aplique en definitiva contemple una disminución de la protección relativa de que gozan los bienes de consumo y un aumento de la que favorece a los bienes de capital y a los insumos intermedios. Si se adopta el AEMC como punto de referencia, se concluye que en bienes de consumo el ajuste mayor con respecto a diciembre de 1973 debe efectuarlo Chile, aunque también Ecuador y Perú tendrán que hacer esfuerzos considerables. En materia de aranceles sobre bienes de capital, los ajustes por parte de estos dos últimos paises serían de mucho menor envergadura que los que debería realizar Chile. ${ }^{58}$

${ }^{85} \mathrm{Los}$ ajustes arancelarios mencionados en el texto sc refieren principalmente a las manufacturas. Cabe destacar que la categoria "bienes de consumo final" in- 
Antes de considerar los efectos probables del AEC sobre cada país, es necesario advertir que su imposición también asume modalidades distintas para los países de menor desarrollo relativo. Disponen éstos, fundamentalmente, de un plazo más extenso para proceder al ajuste, lo cual reduce los costos de transición que la implementación de esta medida involucra. No debe olvidarse la importancia del factor tiempo aun cuando en el análisis que se desarrolla aquí, por razones de espacio no distingamos explícitamente entre efectos en distintos períodos, concentrándonos más bien en los que se suscitan en el mediano plazo.

Estimar la incidencia neta para cada país del movimiento de sus aranceles nacionales hacia el AEC es una tarea muy compleja pues requiere definir previamente un "óptimo" que, en rigor, debería basarse en consideraciones de orden no sólo económico, sino también social. Como es obvio, la imposición del AEC será favorable para un país cuando coincida con el respectivo óptimo nacional, configurándose la situación inversa si las divergencias entre éste y aquél son radicales. Intervienen aquí entonces valores de tipo político-ideológico o apreciaciones sobre las principales distorsiones existentes en la economía, así como juicios acerca de la situación distributiva.

Para eludir el examen de estas variables y concentrarse en los factores económicos, conviene remitirse al análisis de los efectos del AEC, fundándolo en i) las oportunidades de exportar hacia la Subregión que tienen los distintos países a partir de las características de su estructura económica, ii) los cambios que dicho arancel induzca sobre los precios que pagan los consumidores, y iii) la incidencia que genere sobre los ingresos del Estado.

En términos muy gruesos, Bolivia y Ecuador serían los menos beneficiados por la imposición del AEC como tal, puesto que sus aranceles promedios de importación para todos los bienes manufacturados deberían incrementarse. Ellos tendrían que pagar precios superiores por los bienes adquiridos a sus asociados, en circunstancias que estos dos países tienen menores posibilidades de colocar exportaciones en el área andina. El país más afectado, en términos relativos, parece ser Ecuador porque estaría obligado a subir proporcionalmente más sus aranceles sobre bienes de capital ${ }^{59}$-que son

\footnotetext{
cluyc los de origen agrícola. Inicialmente, sin embargo, la mayoría de éstos no se encontraba afccta a aranceles; por el contrario, a menudo gozaba de subsidios. $\Lambda$ cllo sc agrega que los niveles de importación de este tipo de bienes han sido gencralmente elevados, estando la producción doméstica destinada al consumo interno, excepto en el caso de algunos productos específicos, como café, bananos y azúcar.

${ }^{60}$ En cambio, deberí Ecuador reducir o aumentar en un porcentaje mucho menor los aranceles sobre los bicnes de consumo. Uno de los resultados de estos ajustes será la concesión de un menor estimulo relativo para la producción agropecuaria interna, conclusión que también es válida para el caso de Bolivia.
} 
los que, por ausencia de producción interna, debe adquirir casi en su totalidad en el resto del mundo- y enfrenta escasas posibilidades de incrementar sus exportaciones a la Subregión. En cambio, es probable que Bolivia y Ecuador se vean favorecidos con una expansión de sus ingresos arancelarios, sobre todo en la medida en que no sea alta la sustitución entre importaciones subregionales y las provenientes de terceros países. Empero, es previsible que la incidencia neta siga siendo negativa para ambas naciones.

Los países que deberian reducir sus aranceles - en especial Chile y Perú- no estarían necesariamente en una situación inversa, dado que exhiben una estructura económica muy distinta a la de Bolivia y Ecuador. En primer lugar, aunque su protección promedio baje, el nivel de desarrollo de sus industrias les permitiría incrementar sus exportaciones al resto de la Subregión. Por otra parte, ese mismo hecho -que implica un mayor abastecimiento con producción local- significa que las importaciones que ingresan desde el área andina sustituyen más bien producción interna ineficiente antes que abastecimiento proveniente desde terceros países.

La situación relativa del Perú, sin embargo, es menos favorable, tanto por el menor desarrollo relativo del país, como por el hecho particular de que las actividades productoras de bienes de capital se encontraban inicialmente menos protegidas.

Colombia parece ser el país más favorecido con la imposición del AEC. Su nivel promedio no sería muy diferente del vigente antes de la integración. El aumento de la protección relativa de los bienes de capital puede, por otra parte, ser aprovechado por el país para incrementar la producción de ellos, exportando al resto de la subregión ${ }^{60}$. Venezuela está en una situación parecida, aunque no tan evidente. En general, cabría esperar que el ajuste de sus aranceles hacia el probable AEC no tenga efectos muy significativos sobre su economía.

\section{La programación sectorial}

Un problema habitual en todo proceso de integración es que las nuevas industrias privadas que se establecen gracias a la simple liberación de mercados tienden a concentrarse en los países y regiones relativamente más desarrollados dentro del conjunto, debido a que pueden aprovechar la infraestructura física ya instalada, la mejor

${ }^{00}$ Esto pueden conscguirlo mis fícilmente países como Colombia y Chile, en los cuales ya existía alguna producción de csos bienes, pero donde inicialmentc no se estaban aprovechando, dentro de mercados nacionales, toda la capacidad instalada ni las economías de escala disponibles. 
capacitación de la mano de obra, etc. ${ }^{61}$. Los PSDI, por el hecho mismo de impedir que esa localización se produzca en forma "espontánea" según el mercado, favorecen relativamente más a los países de menor desarrollo relativo -Bolivia y Ecuador-, que así pueden incrementar más sus exportaciones hacia el mercado común. También deberían resultar más ventajosos para los países geográficamente localizados en los extremos del Grupo Andino o con mayores dificultades de transporte. ${ }^{62}$

Dado que los PSDI contemplados en el Acuerdo de Cartagena se refieren además a sectores con alta tecnología, grandes plantas y fuertes economías de escala ${ }^{63}$, constituyen un eficiente mecanismo para montar industrias modernas en los países de menor desarrollo relativo que, como muchos, tienden a concebir el crecimiento económico como sinónimo de industrialización. En esta medida, los PSDI son eficientes para distribuir más adecuadamente los beneficios de un proceso de integración.

Por otra parte, en conjunto con la Decisión 24, representan una oportunidad para captar capital extranjero en condiciones más favorables para los intereses de los países. Esto es particularmente importante cuando una de las principales restricciones para lograr un mayor desarrollo es la ausencia en ellos de una capacidad tecnológica y empresarial propia para llevar a cabo nuevas inversiones.

En general, por la racionalización industrial que implica -en especial comparado con la situación inicial en los paises y en un contexto donde la información es tan imperfecta que sc hace difícil que el mercado señale espontáneamente a los productores privados las eventuales ventajas de complementación y especialización-, la programación industrial beneficia a todos los paises. Como es obvio, favorece relativamente más a aquellos que logran una protección arancelaria mayor por los productos que se les asignan, y viceversa. En este sentido, hay que tener siempre en cuenta que las variables mayor producción asignada y menor protección son sustitutivas. De

${ }^{61} \Lambda$ Igunos autores denominan cste fenómeno como el efecto "polarización". Véase especialmente Bird R., "The necds for Regional Policy in a Common Market", 1965, en Robson (ed.), 1972, con respecto al caso de Europa. Este fenómeno fue también observado con mucha nitidez en la ALALC; véase Ffrench-Davis, 1975.

"Bird, op. cit.

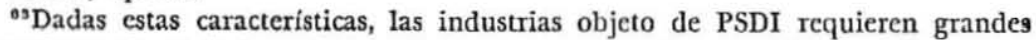
inversiones de capital, razón por la cual este mecanismo también resulta relativamente más favorable para los países que cuentan con volúmencs cuantiosos de ese recurso -Venezuela, por ejemplo-, pues estarán en mejores condiciones de aprovechar realmente los programas que les sean asignados. Sin embargo, esta no es una limitante absoluta, dado que el proceso de integración subregional contempla un mecanismo para contribuir al financiamiento de industrias. Ese es el objetivo de la Corporación Andina de Fomento (CAF). 
allí que ambos aspectos deban ser considerados simultáneamente en la evaluación de los beneficios específicos que obtienen los países de cada programa.

En todo caso los PSDI, en cuanto a mecanismo de integración probablemente tendrían un efecto económico puro relativamente más favorable para los países de menor desarrollo relativo y mayor abundancia relativa de capital.

\section{Regulación común a la inversión extranjera: Decisión 24}

Estimar el efecto económico puro sobre cada país de este mecanismo, crucial en un proceso de integración, resulta mucho más difícil. Se hace patente en este caso, más que en cualquier otro, la importancia del valor subjetivo, político o ideológico, atribuido tanto al instrumento en sí mismo como, implícitamente, a los efectos generales de la inversión extranjera.

Frente a la inversión extranjera (IE), además, esas apreciaciones subjetivas no sólo tienen un origen ideológico, sino también económico. Ellas se plantean en torno a dos cuestiones fundamentales: i) qué alternativas tiene el país para conseguir el aporte real que puede hacer el extranjero (licencias de tecnologias o de marcas registradas, préstamos del capital, inversión de portafolios), o a qué costo podría obtenerse el aporte externo a través de la inversión directa, comparado con el obtenerlo de otras fuentes alternativas; y ii) respecto a qué condiciones y cuánto capital extranjero obtendría un país si no aplicara las políticas comunes que se establezcan.

Una manera de evaluar los efectos que la vigencia de la Decisión 24 tiene para cada país consiste en comparar sus disposiciones con las que regirian internamente en el caso de que aquella no hubiese sido dictada o fuera derogada. Así, por ejemplo, es previsible que en este último evento Perú y Colombia dictarían normas bastantes similares a las que prevalecen en la actualidad para el conjunto de la Subregión, y que Chile las alteraría sensiblemente. Basado en ese criterio, entonces, la conclusión sería que la Decisión 24 es favorable para los primeros y sería juzgada perjudicial por este último. El problema de este método, no obstante, es que en ese juicio estarían interviniendo en forma decisiva preferencias de índole políticoideológica que precisamente deseamos excluir.

No obstante a lo largo de todo este trabajo se ha planteado que para fines analíticos -aunque no en la práctica- es posible separar los aspectos político-ideológicos de los más propiamente económicos. Para concentrarse en estos últimos, conviene distinguir entre la Decisión 24 en cuanto a régimen común igual para todos los países 
vis $\grave{a}$ vis normas nacionales diferentes, y en cuanto a una norma más o menos estricta, pero común, frente al capital extranjero.

En ausencia de programación industrial, las naciones más favorecidas económicamente por este mecanismo en cuanto a norma común serían las de mayor desarrollo relativo. En efecto, con un régimen igual para todos los Estados miembros las inversiones extranjeras para los países -especialmente las dirigidas al sector manufacturero- tenderían a concentrarse en Colombia, Venezuela y Chile, que cuentan con mercados nacionales mayores, una infraestructura más completa, mano de obra más calificada, etc. ${ }^{64}$. Cabe reiterar, sin embargo, que esta sería la situación que se configuraría a igualdad de otras condiciones. En determinados períodos, algunos de los países mencionados podrían recibir menos capital o en condiciones menos ventajosas debido al imperio de condiciones poco auspiciosas en lo político (inestabilidad) o económico (tasa excesiva de inflación, por ejemplo), que los hagan poco atractivos para los inversionistas foráneos.

Desde el punto de vista de la Decisión 24 como un régimen más estricto que el que tenían antes los países, pero común a todos ellos, es probable que tenga una incidencia económica pura menos positiva para las naciones que por diversas razones, de carácter político o económico, contaban inicialmente con menos capital extranjero en relación a su mercado y recursos nacionales disponibles. La razón es que en tal situación probablemente habria margen para absorber un mayor volumen de capital foráneo con una productividad social nacional más elevada que podría hacer un aporte efectivo al desarrollo.

Nuevamente, aquí entran algunos factores subjetivos; no obstante, hay otra forma de plantear el mismo argumento. En efecto, a partir de la norma común de la Decisión 24, que establece que las firmas extranjeras que deseen gozar de liberaciones arancelarias para exportar hacia el mercado ampliado deberán transformarse gradualmente en empresas nacionales, cediendo parte de su activo a inversionistas nacionales, los países que inicialmente tenían mayor capital extranjero en relación al tamaño de su mercado y ahorro nacional se verán relativamente más favorecidos porque existe en ellos más capital extranjero susceptible de ser transferido a nacionales a medida que la integración progrese. Esta sería la situación de Colombia y Venezuela en particular; lo inverso ocurrirá probablementc

${ }^{04}$ En otras palabras, la Dccisión 24 sola (sin PSDI), tiende a equiparar a paises que no son iguales en términos del interés de los inversionistas por radicarse en ellos. La existencia de los PSDI, sin embargo, altera csta situación al obligar a quienes deseen invertir en determinado sector a negociar con el pais que el Grupo decidc. De allí la importancia especial de ambos mecanismos utilizados conjuntamente para las naciones de menor desarrollo relativo. 
con Chile y, en menor medida, con Bolivia y Ecuador. Por este concepto, el primer grupo de naciones estaría más dispuesto a aceptar el régimen común por razones puramente económicas.

Es un hecho demostrado teórica y empíricamente que, sin cambiar las políticas hacia el capital extranjero que los paises tenian antes de la integración, ésta de por sí incrementa la entrada de tales recur$\operatorname{sos}^{65}$. Por lo tanto, dentro de cierto rango, con normas más estrictas podría captarse por lo menos lo mismo (o más) que antes, pero en mejores condiciones ${ }^{60}$. Estas pucden imponerse gracias al mayor poder de negociación conjunta; por lo tanto, la Decisión 24 tendrá per se efectos positivos para todos los países, aunque mayores para unos que para otros.

Evidentemente esos efectos positivos sólo se materializarán si se mantiene una estabilidad mínima de las normas sobre IE.

Existe una estrecha sustituibilidad entre la rigurosidad de las normas frente a las inversiones extranjeras y su inestabilidad, en cuanto al efecto que tienen sobre el volumen de IE que entra a una región. La diferencia crucial entre ambas es que mientras la primera permite captar más beneficios para el país, la inestabilidad permite captar menos beneficios del mismo volumen de inversión, porque el inversionista exige altas rentabilidades para retirar lo más rápidamente posible su capital invertido.

En la práctica, las continuas discusiones sobre la posibilidad de que se cambie la Decisión 24 en el futuro posiblemente ha tenido mayor influencia que la aparente rigurosidad de esa norma en cuanto a no permitir la entrada de mayores capitales que habría sido socialmente beneficiosos para los países recibir. Naturalmente, mientras los inversionistas esperen que en el futuro se relajen las normas vigentes, decidirán postergar su inversión, aun cuando habrían estado dispuestos a hacerlas bajo las condiciones actuales.

Cabe señalar, por último, que Venezuela puede resultar especialmente favorecida, aunque de manera indirecta, con la vigencia de la Decisión 24. En efecto, esta norma discrimina contra las inversiones procedentes del resto del mundo $\mathrm{y}$, por ende, favorece a las que provienen de los Estados miembros, situación que se aprecia con mayor claridad cuando dicha norma es considerada en conjunto con otras Decisiones, como la 46, por ejemplo, relativa a las empresas multinacionales andinas. En este sentido, los nacionales de un

${ }^{65}$ Para un resumen sobre estudios empíricos para el caso del Mercado Común Europeo, véase Tironi (1974).

${ }^{\circ}$ Esto se deriva no sólo de las oportunidades que brinda el mercado ampliado en sí mismo, sino también de la menor competencia entre los países para atraer capital, o la mayor capacidad de negociación conjunta, y la mayor estabilidad de una norma internacional. 
país que como Venezuela dispone de una mayor abundancia relativa de capital tienen la posibilidad de realizar inversiones altamente rentables en el resto del área andina. La Decisión 24 les permite, por ejemplo, adquirir a precios relativamente bajos acciones de propiedad de intereses de fuera de la Subregión, debido a la obligación que estos últimos tienen de transferir parte del capital a nacionales de los países andinos, como única forma de que sus empresas no queden al margen de las ventajas de la integración. ${ }^{67}$

IV. - CONFLICTO Y ARMONÍA DE INTERESES ENTRE LOS PAísES ANDINOS

En el capítulo anterior se ha hecho una estimación de los efectos económicos de los distintos programas de integración sobre cada país. Podría pensarse entonces que bastaría conocer el signo y el valor numérico de cada uno de esos efectos, para determinar la posición global más o menos favorable de cada país frente a la integración andina como un todo, y las perspectivas y problemas que afronta este proceso.

Sin embargo, la afirmación anterior encierra una importante falacia, derivada de la exclusión de los factores político-ideológicos. En efecto, dado que quienes participan en un proceso de integración y toman decisiones son paises independientes, con objetivos propios, la importancia de cada programa no es igual para todos. En otras palabras, no existe un criterio común para percibir ni evaluar esos beneficios; en términos de la teoría económica neoclásica, no existe una función objetivo o de bienestar social aplicable a la Subregión en su conjunto. Por lo tanto, para establecer cuál sería la verdadera posición de cada país es necesario evaluar los efectos económicos de los distintos programas en función de los criterios nacionales relevantes. Estos se encuentran condicionados por valores que hemos denominado político-ideológicos, y que se derivan de la estrategia global de desarrollo.

En este capítulo se integran los factores económicos con los político-ideológicos, a fin de estimar la posición global de apoyo, rechazo o duda (o indiferencia) de cada país respecto del modelo actual de integración andina como un todo y sus programas específicos, cinco años después de iniciado el proceso. Antes de entrar en este

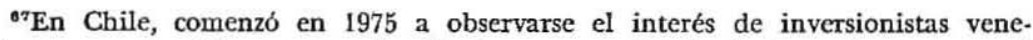
zolanos por adquirir empresas localizadas en aquel país, incluso algunas que el Estado deseaba privatizar. 
análisis es necesario reiterar el carácter aleatorio o circunstancial que pueden tener algunas de tales posiciones.

Ya se mencionó, en primer término, la falta de una definición precisa de las estrategias que siguen los paises. A menudo, ella se manifiesta en inconsistencias entre las declaraciones gencrales y lits aplicaciones prácticas ${ }^{68}$. Otras veces se suscitan evidentes contradicciones entre las recomendaciones concretas de política; por cjemplo, en ocasiones se procura que se establezcan mecanismos generales para estimular las exportaciones de manufacturas hacia terceros paises, mientras se propone un AEC más alto sobre esos bienes.

En segundo término, las actitudes político-ideológicas suelen estar muy influidas en el corto plazo por las posiciones de los gobiernos que en ese momento controlan el Estado. Estos cambian, sin cmbargo, o alteran sus propias posiciones, aunque dentro, normalmente, del margen de maniobra delimitado por los intereses de los grupos sociales dominantes y por la estructura real de la economía. En última instancia, los factores recién mencionados constituyen los rasgos más permanentes que configuran cl patrón de desarrollo que debe seguir el país o los problemas básicos que debe resolver.

En tercer lugar, no debe despreciarse la importancia que tienen las personas mismas que ocupan cargos claves relacionados con la marcha de un país y de la integración. Especialmente en este último campo, la naturaleza de las decisiones que es preciso adoptar exige un grado de conocimiento y una calificación técnica por parte de los representantes de los paises que no siempre existe.

El conjunto de antecedentes político-ideológicos y económicos analizados en los capítulos anteriores ha sido integrado sintéticamente en una matriz de intereses globales de los paises respecto de cada programa (Tabla 1). Ella busca reflejar la actitud de apoyo o rechazo a éstos por razones predominantemente políticas, económicas, o ambas; representaría, por lo tanto, una especic de fotografia de esas actitudes en la actualidad.

En relación a cada programa por separado (es decir, leyendo verticalmente la matriz de intereses) pareciera que en general el Programa de Liberación y los Programas Sectoriales de Desarrollo son los que despiertan menos resistencia o, lo que es lo mismo, los que concitan una mayor armonía de intereses en cuanto a instrumentos de integración. En el caso del primero, los países menos entusiastas serían Ecuador y Bolivia, aunque por la mecánica del modelo andino no tienen tanta razón para preocuparse de sus efectos por el momento. Sus mercados deben empezar a abrirse a las importaciones del resto del área recién a partir de 1976, para concluir en 1986. En

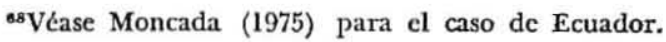


el caso de los PSDI, el apoyo relativamente generalizado al mecanismo como tal no implica ausencia de conflictos particulares en torno a las asignaciones y a los aranceles externos que se determinen para los productos involucrados en ellas.

Las tensiones o divergencias de intereses se concentrarían más marcadamente en torno a la regulación del capital extranjero (Decisión 24) y al AEC. Lo primero ya se ha visto confirmado en la práctica, con Perú y Chile asumiendo las posturas opuestas. En el caso del AEC, las negociaciones están recién comenzando y la experiencia reciente, así como los demás antecedentes analizados en este trabajo, sugieren que van a ser difíciles. Nuevamente, las actitudes más encontradas son las de Chile y Perú, con Colombia y Venezuela en

\section{TABLA 1}

MATRIZ DE INTERESES DE LOS PAÍSES ANDINOS RESPECTO DE LOS DISTTNTOS PROGRAMAS DFL PROCESO DE INTEGRACIÓN SUBREGIONAL

\begin{tabular}{|c|c|c|c|c|c|}
\hline & PL & $\begin{array}{c}\text { AEC } \\
\text { probable }\end{array}$ & PSDI & $\begin{array}{c}\text { Decisión } \\
24\end{array}$ & Total \\
\hline \multicolumn{6}{|l|}{ Estrategia tradicional } \\
\hline Colombia & $A^{\text {el }}$ & $A_{4}$ & $A^{\circ}$ & $A^{01}$ & $\Lambda^{\mathrm{ei}}$ \\
\hline Venczuela & $\mathrm{D}^{\circ}$ & $\mathrm{D}^{\circ}$ & $A^{e 1}$ & $A^{01}$ & $A_{1}-D^{\circ}$ \\
\hline Ecuador & $\mathrm{D}^{\circ}$ & $D^{\circ}-A_{4}$ & $\mathrm{~A}^{\circ}$ & $\mathrm{D}_{1}$ & $\Lambda_{1}-D$ \\
\hline Bolivia & $\mathbf{R}^{\mathbf{e}}$ & $\mathbf{R}^{\circ}$ & $\mathrm{A}^{0}$ & $\mathrm{D}_{1}$ & $D^{\text {o1 }}$ \\
\hline $\begin{array}{l}\text { Estrategia neoliberal } \\
\text { Chile }\end{array}$ & $A^{01}$ & $\mathbf{R}_{1}$ & $D^{e}-R_{i}$ & $\mathbf{R}_{\mathbf{1}}$ & $\mathbf{R}_{1}$ \\
\hline & & & & & \\
\hline $\begin{array}{l}\text { Estrategia planificada } \\
\text { Perú }\end{array}$ & $\mathbf{D}^{\circ}$ & $\mathbf{R}^{\text {e1 }}$ & $\mathrm{A}^{\mathrm{e1}}$ & $\mathbf{A}_{\mathbf{1}}$ & $D^{e 1}$ \\
\hline
\end{tabular}

Simbolos: $\mathbf{A}=$ Apoyo; $\mathbf{D}=$ Duda o indiferencia; $\mathbf{R}=$ Rechazo.

Subindices: $\mathrm{c}=$ por razones predominantcs económicas; $\mathrm{i}=$ por razones ideológicas o valorativas (determinadas por la respectiva estrategia de desarrollo) .

Nota: El subrayado bajo determinadas posiciones en la matriz indica que de acuerdo a la respectiva estrategia nacional de dcsarrollo para el pais de que se trata ese programa o política tiene la más alta prioridad. Por ejemplo, los PSDI dentro de la estrategia planificada (Perú) y el AEC dentro de la ncoliberal (Chile). 
posiciones intermedias (éste último más cerca del segundo y viceversa) que pueden ser muy decisivas para inclinar la balanza en uno $u$ otro sentido. ${ }^{69}$

Es evidente que cada pais no puede esperar ser el más favorecido por la aplicación de cada uno de los programas o mecanismos. Como se ha destacado desde el comienzo, la integración implica un compromiso en diversos planos. Las naciones que la impulsan deben estar dispuestas a sacrificar ciertos beneficios o a incurrir en ciertos costos -económicos o políticos- para asegurar las ventajas netas positivas que el proceso ha de generar si continúa adelante.

La posición global de cada país frente a la integración subregional depende tanto de los efectos económicos puros de los distintos programas básicos - tal como se han ido definiendo hasta 1975-, como de la prioridad que se les asigne en función de las estrategias nacionales de desarrollo.

En una sintesis muy apretada, los países que han seguido una estrategia de tipo tradicional parecen apoyar de manera relativamente más decidida la integración andina en la forma concreta que ésta ha asumido hasta ahora. Para ellos tendrían similar prioridad una política arancelaria que involucrara una protección razonable a la producción interna (AEC intermedio) y una programación sectorial compatible con esas condiciones arancelarias. Distinguiendo más entre ellos, los PSDI alcanzarían comparativamente una mayor ponderación en los países de menor desarrollo relativo. Colombia, por su parte, apoyaría de manera más decidida el proceso de liberación misma y un AEC más bajo, principalmente por razones derivadas de factores económicos estructurales obvios, antes que por consideraciones de carácter politico-ideológico.

Perú, que sigue una estrategia de desarrollo más planificado, se encuentra sometido a fuertes tensiones: por una parte, cabe esperar que brinde un apoyo decidido a la Programación Sectorial y a la Decisión $24 \mathrm{y}$, por otra, que manifieste un vigoroso rechazo a las proposiciones de la mayoría de los países sobre un AEC que considera muy bajo, y dudas respecto a un PL que se desarrolle en esas condiciones. Su posición global, en consecuencia, resulta más difícil de establecer. Ello obedece fundamentalmente a que el modelo peruano ha evolucionado en una dirección bastante diferente que la que estaba implícita en el proceso de integración subregional. Así, por ejemplo, por la importancia que busca concederle a la propiedad estatal de empresas estratégicas, ese país necesita la programación sec-

${ }^{\circ}$ Véanse los informes del Primer y Segundo Consejo de Comercio Exterior del Grupo Andino, JUNAC (1975 d y e). También discusiones en la prensa a propósito de la reunión de los Ministros de Hacienda del Grupo Andino en Colombia, entre el 6 y 8 de diciembre de 1975 . 
torial, y en parte por esas mismas razones, el sector privado interno se resistiria a realizar las inversiones requeridas para aprovechar las ventajas del PL.

En el caso de Chile, que actualmente sigue una estrategia de tipo neoliberal o de libre comercio, uno de los programas básicos que condicionaría su apoyo a la integración es la política arancelaria externa y, en menor medida, la Decisión 24. En este sentido, su disconformidad con el AEC que probablemente proponga la Junta, por considerarlo demasiado alto, estaría implicando un virtual rechazo al proceso como un todo. Esta actitud refleja una posición según la cual el incentivo para el desarrollo del comercio con la Subregión no debe ser mucho más fuerte que aquel para el desarrollo del consumo con el resto del mundo. En la medida que tal actitud deriva de una estrategia global de desarrollo divergente con la de los demás países, decimos que proviene de consideraciones políticoideológicas diferentes. Esto se hace más patente si se piensa que, mirado desde el punto de vista del Grupo Andino, Chile es, en términos comparativos, uno de los países que más podría beneficiarse económicamente con Ia integración, en especial por sus posibilidades de exportar manufacturas a través del PL. Cabe recalcar que esta circunstancia, unida al hecho de que colocar exportaciones de productos elaborados en terceros países es más difícil que hacerlo en la Subregión, puede llevar a que los sectores empresariales presionen por un gradual cambio de esa estrategia en el futuro, haciendo predominar finalmente una visión más pragmática, que reconozca las limitaciones y distorsiones del mercado internacional de manufacturas.

\section{v. - PERSPECTIVAS DEL PACTO ANDINO}

La conclusión básica que se desprende de este análisis es que los principales conflictos de intereses que se suscitan entre los países andinos se originan en una diferente percepción sobre las alternativas que ellos tienen para desarrollarse; es decir, son resultado de las diferentes estrategias que siguen.

En este contexto es más útil y relevante comprender las divergencias de tipo político o de carácter ideológico que han aparecido a través del tiempo. En efecto, al cabo de cinco años de haberse iniciado el proceso de integración andino algunos países aparecen impulsando estrategias de desarrollo bastante distintas de las que se 
habían trazado cuando, a fines de la década del 60 , se formularon las bases del esquema que se continúa aplicando, por corresponder a los compromisos contemplados en el Acuerdo de Cartagena.

También el contexto internacional se ha visto modificado en el transcurso de estos años, siendo necesario en consecuencia considerar explícitamente los efectos visibles o virtuales de esos cambios. Reconocer e identificar con más precisión la naturaleza de éstos es lo primero que hay que hacer para establecer las perspectivas del proceso y determinar las medidas que se requiere adoptar para que siga avanzando con el dinamismo observado hasta ahora.

Las perspectivas de la integración andina son muy difíciles de anticipar. El análisis al respecto podría plantearse desde dos ángulos diferentes. El primero de ellos puede resumirse en la pregunta acerca de cuáles son las posibilidades de que cada país decida permanecer en el Grupo si éste mantiene las características básicas que ha exhibido hasta ahora. La otra forma de encarar el problema se refiere a cómo deberían ajustarse los programas o mecanismos contemplados en el Acuerdo para que todos los países estuviesen dispuestos a permanecer en el Grupo. Ambas perspectivas constituyen, es cierto, planteamientos extremos; sirven, no obstante, para ilustrar las perspectivas básicas del proceso. La primera pregunta es poco realista porque todo acuerdo - especialmente el andino, por la forma como fue concebido- supone que varias de sus características o programas puedan ir ajustándose en el transcurso del tiempo. Esa interrogante, sin embargo, sirve para ilustrar cuál es el último recurso de negociación de un pais, que sería retirarse completamente del Pacto si sus intereses respecto de uno o más programas que estima prioritarios entran en conflicto con los del conjunto de sus asociados. La segunda interrogante sería más realista en la medida que los intereses entre países no divergieran sustancialmente. Para estimar cuáles, cómo y en qué sentido deberían ajustarse los diversos programas básicos de la integración andina habría que conocer el poder de negociación relativo de los Estados, aparte de los intereses político-ideológicos y económicos de cada uno. Ese poder estará dado principalmente por las alternativas que tengan los países para crecer o industrializarse y por la magnitud del mercado que puede ofrecer a sus demás socios, es decir, por su tamaño económico. Así, por ejemplo, el peso del eventual alegato de Ecuador en contra del AEC será mucho menor que el de Venezuela, que está ofreciendo un mercado potencial cuatro veces más grande.

Sin entrar en ese tema de por sí muy complejo y que obligaría a introducir nuevos elementos de análisis, conviene plantear el problema en términos de las principales concesiones o sacrificios de ciertos intereses nacionales que debe hacer cada Estado si se desea 
que la integración andina continúe con las características básicas que la han definido hasta ahora. Esto implica concentrar el peso del ajuste al interior de cada país, de modo de sustituir los efectos percibidos como "desfavorables" de un programa, por una parte de los positivos derivados de otros. En tal sentido, parecería en general más lógico esperar que los ajustes principales proviniesen de los países que siguen estrategias de desarrollo más divergentes con respecto a la que dio origen al actual modelo de integración, que son Ghile y Perú. En particular, esto requeriría que el primero estuviese dispuesto a aceptar el actual Régimen Común de Tratamiento al Capital Extranjero, a cambio de un AEC relativamente más bajo que el que desean los demás países. Perú, en cambio, debería aceptar un AEC más bajo, a cambio de un mayor énfasis en los PSDI o una protección más alta para ellos.

En el caso del resto de los países, las concesiones necesarias serían menos significativas, debido a que sus intereses nacionales no difieren tanto de aquellos que reflejan el consenso básico ya definido en el Acuerdo de Cartagena. Es cierto que Bolivia y Ecuador plantean algunas discrepancias importantes en torno al AEC, pues desearían que fuera inferior a lo proyectado, especialmente en lo tocante a los bienes de capital, y otras más bien implícitas con respecto al PL que se aplicaría dentro de ese marco arancelario. Sin embargo, a cambio de eso, tienen las asignaciones de PSDI, las cuales pueden compensar con creces los efectos anteriores menos positivos, aparte de todo el tratamiento preferencial sobre modalidades del PL y plazos para adoptar el AEC.

Lo importante, sin embargo, es no alarmarse porque se susciten conflictos ni apresurarse a deducir de allí que todo el proceso está en crisis. La clave está en que existan, en primer lugar, los mecanismos e instituciones que permitan resolver las contradicciones, en función de criterios claros. Esta es precisamente una de las principales cualidades del esquema andino, que ha sido demostrada en diversas oportunidades, pero que es, sin duda, susceptible de perfeccionarse.

En segundo lugar, resulta imprescindible que cada país tenga conciencia de que la integración se logra mediante la aplicación de un conjunto de mecanismos o programas comunes y no sólo de los que se ajustan más a sus intereses nacionales. Además, todos aquellos tienen efectos positivos y negativos que son intercambiables.

Lamentablemente, la visión de conjunto de los distintos programas no es fácil cuando cada uno debe negociarse en forma aislada debido a la imposibilidad operativa de hacerlo de manera simultánea. El problema se agudiza cuando es difícil hacer estimaciones relativamente confiables de los efectos económicos puros (positivos 
o negativos) de cada programa y cuando la "valoración" de ellos en las estrategias nacionales de desarrollo es poco explícita y varía mucho con el transcurso del tiempo. En este sentido, el estudio de tales efectos y, particularmente, de la distribución de beneficios de la integración entre países resulta indispensable.

En última instancia, sin embargo, no es posible desconocer que la integración entre los países andinos será más completa y beneficiosa para todos en la medida que se vaya perfilando una estrategia de desarrollo común o similar para el conjunto de naciones. Esa estrategia debe, además, responder efectivamente a las necesidades y características más profundas de los países y tener una estabilidad mínima para que pueda rendir sus frutos. En este sentido, la comprobación de las ventajas y efectividad real de un proceso de integración no depende sólo de su "éxito" o "fracaso" en términos de su avance mediante la implementación de las medidas acordadas, sino también de que toda ella responda a una estrategia de desarrollo común que permita resolver los problemas fundamentales y urgentes de los pueblos andinos.

\section{REFERENCIAS BIBLIOGRAFICAS}

Aninat, A. (1975), "El programa de liberación y el arancel cxterno común en cl Acuerdo de Cartagena", mimeo, CINDA.

Avila, M. (1974), "Programación de la industria metalmecánica en el Acuerdo de Cartagena", Revista de la Integración, No 13, INTAL, Buenos Aircs.

Caballero, C. (1975), "El desarrollo económico colombiano y la integración andina", mimeo, CINDA.

Camacho, E. (1975), La integración andina: fundamentos politicos y perspectivas, Ed. Los Amigos del Libro, La Paz.

CEPAL (1974), "Algunas conclusiones relativas a la integración, la industrialización y el desarrollo económico en América Latina", Boletin Económico para América Latina, vol. XIX, No 1 y 2.

CINDA, et al. (1973), Variables politicas de la integración andina, Ed. Nucva Universidad, Santiago de Chile.

Ffrench-Davis, R. (1973), "La integración andina", Revista de la Universidad Técnica del Estado, No 11 - 12, Santiago de Chile.

- (1974, "La planificación en el Pacto Andino y el Arancel Externo Común", Revista de la Integración, No 17, INTAL, Buenos Aires.

- (1975), "El Pacto Andino" un modelo original de integración", Estudios de Planificación, № 42, CEPLAN, Santiago de Chile.

INTAL (1975), El proceso de integración en América Latina en 1974, Bucnos Aires.

Iñíguez, V. y Vega-Centeno, M. (1975), "El modelo peruano de desarrollo y la integración", mimeo, CINDA.

Corden, W. M. (1972), "Economic of scalc and customs union theory", Journal of Political Economy, Vol. 80, Ne 3, mayo/junio. 


\section{ESTUDIOS INTERNACIONALES}

Isla, E. y Campos, J., Acuerdos de complementación: su aprovechamiento por las empresas nacionales", Revista de la Integración, No 17, INTAL, Buenos Aircs.

Jaber, T. (1971), "La importancia de la teoría tradicional de la integración para los países subdesarrollados", Revista de la Integración, № 9, INTAL, Buenos Aires.

JUNAC (1974), "Informe final del primer seminario sobre planificación integrada de los países miembros". JUN/SI. PL./I, Lima, diciembre.

(1975) a), "Proyecto de bascs para la elaboración del arancel externo común", CCE/II/dt 14, Lima, abril.

- (1975 b), "Aplicación del criterio del empleo en el arancel externo común", CCE/II/dt 9, Lima.

(1975 c), "El nivel del arancel externo común", mimeo, CCE/II/dt 12,

Lima, mayo.

(1975 d), "Informe final de la segunda reunión del Consejo de Comercio Exterior", CCE/II, Santiago de Chile, septiembre.

- $(1975 \mathrm{c})$, "Informe fiscal de la tercera reunión del Consejo de Comercio Exterior", Lima, diciembre.

Lipsey, S. (1960), "The theory of customs unions: a general survey", Economic Journal, Vol. LXX, № 279, septiembre. También en Caves y Johnson (eds.), Reading in International Economics, Irwin, 1968, U.S.A.

Lizano, E. (1973), "Problemas actuales de la integración: la distribución de beneficios y costos de la integración entre países en desarrollo", mimeo, $\mathrm{Na}$ ciones Unidas, $\mathrm{TD} / 8 / 394$.

- (1974), "La distribución de beneficios y costos de la integración económica, un procedimiento alternativo", Revista de la Integración, vol. VII, Ne 15, Buenos Aires, enero.

Lizano, E. y Wilmore, L. (1975), "La integración económica de Centroamérica y el Informe Rosenthal", El Trimestre Económico, No 165, encro - marzo.

Melo, M. (1973), "La integración subregional andina, el punto de vista colombiano", mimeo, Centro de Investigaciones para el Desarrollo, Universidad Nacional de Colombia, Bogotá.

Moncada (1975), "El desarrollo económico del Ecuador y la integración andina", mimeo, CINDA.

Poulantzas, N. (1970), Poder politico y clases sociales en el Estado capitalista, Siglo XXI Editores, México.

Robson, P. (1972), International economic integration (Ed.), Penguin Books, England.

Salgado, G. (1973), "El Grupo Andino y la inversión extranjera", Comercio Exterior, Vol. XXIII, México, febrero y mayo.

Tironi, E. (1974), "Determinants of direct foreign investment: an econometric study of US capital in Latin Amcrican manufactures", mimeo, CEPLAN, Santiago de Chile.

Tironi, E. (1975), "Aspectos teóricos del comportamiento de corporaciones transnacionalcs frente a un proceso de integración", Estudios de Planificación, No 48, CEPLAN, Santiago de Chile y Revista de la Integración, No 19/20, mayo-sept. 